\title{
Dynamical evolution of planetary systems
}

\author{
Alessandro Morbidelli \\ Dep. Cassiopee, University of Nice - Sophia Antipolis, CNRS, Observatoire de la Côte d'Azur; Nice, France
}

\begin{abstract}
The apparent regularity of the motion of the giant planets of our solar system suggested for decades that said planets formed onto orbits similar to the current ones and that nothing dramatic ever happened during their lifetime. The discovery of extra-solar planets showed astonishingly that the orbital structure of our planetary system is not typical. Many giant extra-solar planets have orbits with semi major axes of $\sim 1$ AU, and some have even smaller orbital radii, sometimes with orbital periods of just a few days. Moreover, most extra-solar planets have large eccentricities, up to values that only comets have in our solar system. Why such a big diversity between our solar system and the extra-solar systems, as well as among the extra-solar systems themselves? This chapter aims to give a partial answer to this fundamental question. It's guideline is a discussion of the evolution of our solar system, certainly biased by a view that emerges, in part, from a series of works in which I have been involved. According to this view, the giant planets of the solar system suffered radial migration while they were still embedded in a proto-planetary disk of gas and presumably achieved a multi-resonant orbital configuration, characterized by smaller inter-orbital spacings and smaller eccentricities and inclinations with respect to the current configuration. The current orbits of the giant planets have been achieved during a phase of orbital instability, during which the planets acquired temporarily large-eccentricity orbits and all experienced close encounters with at least another planet. This instability phase occurred presumably during the putative "Late Heavy Bombardment" of the terrestrial planets, approximately 3.9 Gy ago (Tera et al., 1974). The interaction with a massive distant planetesimal disk (the ancestor of the current Kuiper belt) eventually damped the eccentricities of the planets, ending the phase of mutual planetary encounters and parking the planets onto their current, stable orbits. This new view of the evolution of the solar system, makes our system not very different from the extra-solar ones. In fact, the best explanation for the large orbital eccentricities of extra-solar planets is that the planets that are observed are the survivors of strong instability phases of original multi-planet systems on quasi-circular orbits. The main difference between the solar system and the extra-solar systems is in the magnitude of such an instability. In the extra-solar systems, encounters among giant planets had to be the norm. In our case, the two major planets (Jupiter and Saturn) never had close encounters with each other: they only encountered "minor" planets like Uranus and/or Neptune. This was probably just mere luck, as simulations show that Jupiter-Saturn encounters in principle could have occurred. Another relevant
\end{abstract}


difference with the extra-solar planets is that, during the gas-disk phase, our planets avoided to migrate permanently into the inner solar system, thanks to the specific mass ratio of the Jupiter/Saturn pair and the rapid disappearance of the disk soon after the formation of the giant planets. This chapter ends on a note on terrestrial planets. The structure of a terrestrial-planet system depends sensitively on the dynamical evolution of the giant planets and on their final orbits. It appears clear that habitable terrestrial planets, with moderate eccentricity orbits, cannot exist in systems where the giant planets became violently unstable and developed very elliptic orbits. Thus, our very existence is possible only because the instability phase experienced by the giant planets of our solar system was of "moderate" strength.

\section{Introduction}

It is now clear that the planetary systems that we observe did not form in their current configuration, but they have been heavily modified by a non-trivial dynamical evolution. Processes like planet migration, resonant trapping, planet-planet scattering, mutual collisions, hyperbolic ejections have sculpted the structure of planetary systems since the formation of the planets; some of these processes might also have played a crucial role in the accretion of the planets themselves. The observational evidence that planetary systems can be very different from each other, suggests that their dynamical evolutions have been very diverse, probably as a result of a strong sensitivity of the dynamics on environmental parameters or initial conditions.

The goal of this chapter is to review our current understanding of the possible dynamical evolutions of planetary systems and of the open problems that we face. The main focus will be on our solar system, because this is the system that we can best model, thanks to the vast number of observational constraints. We will see that the dynamical history of our planetary system followed step-wise generic processes, but was also characterized by a number of specific "events" that act like bifurcation points in the evolution of planetary systems. I will then discuss what would have happened if these events had occurred differently. This will give us some guidance in understanding the origin of the diversity of planetary systems.

This chapter is divided in three parts. The first is devoted to the early evolution of giant planets when they are still embedded in the gas-disk, i.e. during the first few millions of years following the formation of the central star. The second discusses the evolution of the giant planets after the disappearance of the gas, when they interact with a still massive planetesimal disk; this is the era of debris disks, which are commonly observed around stars even as old as 1Gy. The third part will focus on terrestrial planets and on how their accretion and evolution depend on the evolution of the giant planets discussed before. 


\section{The gas-disk era}

\subsection{The formation of the giant planets}

There are two possible mechanisms by which we envision that giant planets can form. The first is nicknamed the "core-accretion mechanism": the coagulation of solid particles forms a core typically of about 10 Earth masses $\left(M_{\oplus}\right)$ while the gas is still present in the proto-planetary disk; the core then traps by gravity a massive atmosphere of hydrogen and helium from the disk (Pollack et al., 1996) and becomes a giant planet. The second mechanism invokes the gravitational instability of the gaseous component of the disk (Cameron, 1978): a cold, massive proto-planetary disk can break into a number of self-gravitating gas-clumps, which then contract forming giant gaseous planets (Cassen et al., 1981; Boss, 2000, 2001, 2002; see Durisen et al., 2007 for a review).

The debate to discriminate between these two models has been very intense over the last 10 years. Now, several direct or indirect observations suggest that the core-accretion mechanism is predominant for the formation of the planets detected so far. First, interior structure models of the giant planets of the solar system predict that all of them have massive solid cores (Guillot, 2005; Militzer and Hubbard, 2009; however see Nettelmann et al., 2008 for a model arguing for a core-less Jupiter). Second, there is a clear correlation between the metallicity of stars and the probability that said stars have giant planets around them (Fisher and Valenti, 2005). Third, transiting extra-solar planets are inferred to have solid cores whose relative mass is correlated with the metallicity of the host star (Guillot et al., 2006). All these features suggest that solids have a crucial role in giant planet formation, an aspect that is difficult to explain in the framework of the gravitational instability model (Boss, 2002). Moreover, new hydro-dynamical simulations which model more accurately the thermodynamics in the proto-planetary disk find that formation of long-lived self-gravitating clumps of gas is likely only at large distances from the central star (> 50-100 AU; Boley, 2009). It is still unclear, though, whether the end-products of these clumps can be giant planets or must be brown-dwarf-mass objects (Stamatellos and Whitworth, 2008).

Thus, there is a growing consensus that the giant planets observed within a few AUs from their parent stars formed by the core-accretion process. The planets found at large distances from their parent stars (for instance around HR 8799 - Marois et al., 2008- or Fomalhaut - Kalas et al., 2008) are the best candidates to be the outcome of the gravitational instability process. There is still a possibility, though, that they are giant planets formed closer to the star by core-accretion, which subsequently achieved large orbital distances through planet-planet scattering (Veras et al., 2009) or outwards migration (Crida et al., 2009). I will return to this, further down in the chapter.

The core-accretion model, nevertheless, has its own problems. The main difficulty is to understand how a $\sim 10 M_{\oplus}$ core could form within a few million years (which is the typical survival time of a gas disk; Haisch et al., 2001). In the classical view, these cores form by collisional coagulation from a disk of planetesimals (small bodies of sizes and compositions similar to current asteroids or comets). In this environment, gravity starts to play a fundamental role, bending the trajectories of 
the colliding objects; this leads to an effective increase of the collisional cross-section of the bodies by the so-called gravitational focussing factor (Greenzweig and Lissauer, 1992). At the beginning, if the planetesimal disk is dynamically very cold (i.e. the orbits have tiny eccentricities and inclinations), the dispersion velocity of the planetesimals $v_{\text {rel }}$ may be smaller than the escape velocity of the planetesimals themselves. In this case, a process of runaway growth begins, in which the relative mass growth of each object is an increasing function of its own mass $M$, namely:

$$
\frac{1}{M} \frac{\mathrm{d} M}{\mathrm{~d} t} \sim \frac{M^{1 / 3}}{v_{\mathrm{rel}}^{2}}
$$

(Greenberg et al., 1978; Wetherill and Stewart, 1989). However, as growth proceeds, the disk becomes dynamically heated by the scattering action of the largest bodies. When $v_{\text {rel }}$ becomes of the order of the escape velocity from the most massive objects (i.e. $v_{\text {rel }} \propto M_{\text {big }}^{1 / 3}$ ), the runaway growth phase ends and the accretion proceeds in an oligarchic growth mode, in which the relative mass growth of the largest objects $\mathrm{d} \log M_{\mathrm{big}} / \mathrm{d} t$ is proportional to $M_{\mathrm{big}}^{-1 / 3}$ (Ida and Makino, 1993; Kokubo and Ida, 1998).

In principle, the combination of runaway and oligarchic growths should continue until the largest objects achieve an isolation mass, which is a substantial fraction of the initial total mass of local solids. In the outer Solar System, beyond the so-called snowlin 1 (Podolak and Zucker, 2004), if the initial disk is sufficiently massive (about 10 times the so-called Minimal Mass Solar Nebula or MMSN; Weidenschilling, 1977; Hayashi, 1981) it is expected that the end result is the formation of a few super-Earths (Thommes et al., 2003; Goldreich et al., 2004; Chambers, 2006), as required in the core-accretion model for giant planet formation. $N$-body simulations, though, show that reality is not so simple. When the cores achieve a mass of about $1 M_{\oplus}$ they start to scatter the planetesimals away from their neighborhood, instead of accreting them (Ida and Makino, 1993; Levison et al. 2010), which slows their accretion rate significantly. It has been proposed that gas drag (Wetherill and Stewart, 1989) or mutual inelastic collisions (Goldreich et al., 2004) prevent the dispersion of the planetesimals by damping their orbital eccentricities, but in this case the cores open gaps in the planetesimal disk (Levison and Morbidelli, 2007; Levison et al., 2010), like the satellites Pan and Daphis open gaps in Saturn's rings. Thus the cores isolate themselves from the disk of solids. This effectively stops their growth. It has been argued that planet migration (Alibert et al., 2004) or the radial drift of small planetesimals due to gas drag (Rafikov, 2004) break the isolation of the cores from the disk of solids but, again, $N$-body simulations show that the relative drift of planetesimals and cores simply collects the former in resonances with the latter (Levison et al., 2010); this prevents the planetesimals from being accreted by the cores.

In summary, the accretion of massive cores is still an open problem, and I am suspicious of synthetic models where simple formulæ for the mass-growth of the cores are made up to achieve

\footnotetext{
${ }^{1}$ The orbital radius beyond which temperature is cold enough that water condenses into ice. The snowline is situated at about 3-5 AU from a solar-mass star, depending on time and on disk models (Min et al., 2011).
} 
the desired result, without any correspondence with the outcomes of $N$-body simulations (which should be considered as a sort of "ground truth" for dynamical processes).

Another problem of the core-accretion model originates from Type-I migration. Type-I migration is the label denoting the radial drift of planetary cores (with masses ranging from that of Mars to several Earth masses) due to their gravitational interaction with the gaseous component of the disk. Analytic and numerical studies have shown that a planetary core generates a spiral density wave in the disk (Goldreich and Tremaine,1979,1980; Ward, 1986, 1997; Tanaka et al. 2002). In the outer part of the disk, the wave trails the core. Thus, the gravitational attraction that the wave exerts on the core results in a negative torque that slows the core down. In the inner part of the disk, the wave leads the core, and therefore it exerts on it an acceleration torque. The net effect on the core depends on the balance between these two torques of opposite signs. Ward (1997) showed that in general cases, i.e. for disks with power-law radial density profiles, the negative torque exerted by the wave in the outer disk wins. Consequently the core has to lose angular momentum, and its orbit shrinks: the planetary core migrates towards the central star, with a speed:

$$
d a / d t \propto M_{p} \Sigma_{g}(a / H)^{2},
$$

where $a$ is the orbital radius of the planet, $M_{p}$ is its mass, $\Sigma_{g}$ is the surface density of the gas disk and $H$ is its height at the distance $a$ from the central star.

Precise calculations show that an Earth-mass body at 1 AU, in a MMSN with scale height $H / a=5 \%$, migrates into the star in $200,000 \mathrm{y}$. Thus, planetary cores should fall onto the central star well before they can attain the mass required to capture a massive atmosphere and become giant planets.

Several mechanisms that might weaken or prevent Type-I migration have been investigated. First, turbulence may turn inward Type-I migration into a random walk (Nelson and Papaloizou, 2003; Nelson, 2005), which could save at least some of the cores. Second, if there is a steep positive $d \Sigma_{g} / d a$ at some location in the disk, for instance at the outer edge of a partially depleted central cavity, inward Type-I migration should stop there (Masset et el. 2006). Finally, it has been recently shown that migration can be outwards in the inner part of the disk, which transports and dissipates heat inefficiently due to its large opacity (Paardekooper and Mallema, 2006; Baruteau and Masset, 2008; Kley and Crida, 2008; Paardekooper et al., 2010). In this case, all cores would migrate towards an intermediate region of the disk, where Type-I migration is effectively erased (Lyra et al., 2010).

A particularly puzzling aspect of the core-accretion process is the evidence that in our solar system massive cores of $\sim 10 M_{\oplus}$ formed in the giant planets region, whereas in the inner solar system the planetary embryos resulting from the runaway/oligarchic growth process presumably had masses smaller than the mass of Mars (Wetherill and Stewart, 1993; Wetherill, 1992; Weidenschilling et al., 1997). This jump of two orders of magnitude in the masses of embryos/cores from the inner to the outer solar system is difficult to understand. In fact, the surface density of solids in the disk should have had a 'jump' at the snowline of only a factor of $\sim 2$, given the revised solar $\mathrm{C} / \mathrm{O}$ 
abundance (Lodders, 2003). Moreover, the orbital frequency (that sets the speed of all dynamical processes, including accretion) decreases with increasing distance from the star. So, what makes the outer solar system so favorable for the formation of massive cores? To answer this question, several investigators searched for mechanisms that can concentrate a large amount of solids (well above the initial surface density) in some localized region of the disk, so to achieve a sweet spot for the formation of a massive object. Some proposed mechanisms still give a pivotal role to the snowline (Morfill and Volk, 1984; Ida and Lin, 2008), but others are based on the concentration of boulders in long-lived vortices (Barge and Sommeria, 1995; Lyra et al., 2009a, 2009b), or on halting migration of planetary embryos at a given orbital radius (Masset et al., 2006; Morbidelli et al., 2008a; Paardekooper and Papaloizou, 2009; Sandor et al, 2011), which are independent of the snowline location. Obviously more work is needed to understand which mechanism is relevant and dominant. Depending on future results, it might turn out that the wide-spread expectation that giant planets form by the core-accretion mechanism only beyond the snowline is naive; instead, some giant planets might have formed in the warmer regions of the disk (Bodenheimer et al., 2000).

\subsection{Once giant planets are formed: Type II migration and its consequences}

By the action-reaction principle, the torques exerted by the disk onto the planet are symmetrically exerted by the planet onto the disk. Thus, the planet exerts a positive torque onto the outer disk, i.e. it pushes the outer disk outwards, while it exerts a negative torque onto the inner disk, i.e. it pushes it inwards. For small mass objects, such as the planetary cores considered in the previous section, these torques are overcome by the internal torques of the disk, due to viscosity and pressure, so that the mass distribution in the disk -averaged over the azimuthal coordinate- is not significantly affected by the presence of the planet. However, this is no longer true if the planet is sufficiently massive (several tens of Earth masses for typical values of the disk's parameters); in this case the internal torques of the disk cannot oppose the torques suffered from the planet. Consequently, the inner and outer parts of the disk are effectively repelled, and a gap in the gas distribution opens around the planet's orbit (Lin and Papaloizou, 1986a; Crida et al., 2006).

Once a giant planet has opened a gap in the disk, it is condemned to stay in the middle of the gap. In fact, if it approached the inner edge of the gap, the distance of the planet from the inner disk would decrease and that from the outer disk would increase, so that the torque felt from the inner disk would become stronger than that felt from the outer disk. Thus the planet would be pushed back towards the center of the gap. The symmetric situation would occur if the planet approached the outer edge of the gap. Consequently, any radial migration of the planet has to follow the radial migration of the gap. The global evolution of the disk occurs on a viscous timescale $T_{\nu}=a^{2} / \nu$, where $\nu$ is the disk viscosity (Lynden-Bell and Pringle, 1974). So, the radial displacement of the gap and the migration of the planet have to occur on this timescale (Lin and Papaloizou, 1986b). Unless the planet is close to the outer edge of the disk (Veras and Armitage, 2004), the planet has to migrate towards the star, because this is the natural direction of evolution 


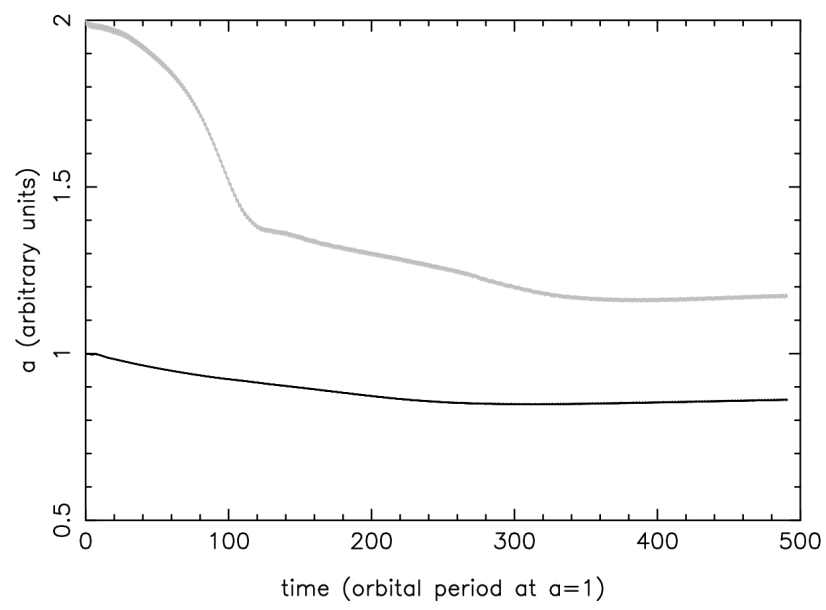

Fig. 1.- An illustration of the dynamical evolution of Jupiter and Saturn in the gas-disk, as in Masset and Snellgrove (2001). The black and grey curves show the evolutions of the semi major axes of Jupiter and Saturn, respectively. Capture in the $2 / 3$ mean motion resonance occurs when the migration of Saturn is reversed.

of an accretion disk (Lynden-Bell and Pringle, 1974; Lin and Papaloizou, 1986b). The migration of a planet associated with a gap is called "Type II migration".

Type II migration explains in a natural way the presence of giant planets on orbits very close to the parent stars (Lin et al., 1996), which is a very common characteristic in the population of extra-solar planets discovered to date. However, in our solar system the giant planets have orbital radii of several AUs, comparable to the orbital radii at which said planets are expected to have formed (see sect. 2.1). Moreover, several extra-solar planets have also been discovered on orbits with semi major axes larger than $\sim 3$ AU. What happened in these cases? Why is Type II migration sometimes ineffective?

For our solar system, the key to answer this question seems to be the co-existence of Jupiter and Saturn, with their specific mass ratio. In fact, hydro-dynamical simulations, where Jupiter and Saturn are simultaneously taken into account with fixed masses, show that Saturn migrates the fastest, due to it smaller mass; therefore, it has no trouble in approaching Jupiter until the two planets are caught in resonance (Masset and Snellgrove, 2001). In disks with mass comparable to the MMSN, the most likely end-state is the capture in the mutual $2 / 3$ mean motion resonance, where the orbital period of Saturn is 1.5 that of Jupiter. This occurs even if Saturn is initially beyond the $1 / 2$ resonance or locked into the $1 / 2$ resonance (Pierens and Nelson, 2008). Stable capture into the $1 / 2$ resonance is possible only for disks with surface density decaying less steeply than $1 / \mathrm{r}$ or in low-mass disks (Zhang and Zhou, 2010). Once locked in the $2 / 3$ resonance, the inward migration of Jupiter and Saturn stops (Masset and Snellgrove, 2001; Morbidelli and Crida, 2007), which explains why Jupiter did not migrate all the way close to the Sun. These results hold both for disks with constant viscosity and for the so-called $\alpha$-disks (Shakura and Surayev, 1973) and do not depend critically on the value of the viscosity. 
However, things may not be so simple in reality. All the hydrodynamical simulations that show that Saturn captures Jupiter in resonance, assume fixed masses for the planets. A natural question arises: is it still reasonable to expect resonance capture if the migration histories of the planets are coupled with their accretion histories? At first sight the answer is negative. If the second planet forms later than the first one, its migration history should just replicate that of the first planet, but later in time. More simply, the second planet should always lag behind the first one, as it is just repeating the evolution of the first planet, just at a later time. Thus, it appears that Saturn could catch Jupiter in resonance only if the accretion histories of the two planets were different. In particular, if Jupiter grew very rapidly to its current mass, it would have passed very quickly to a Type-II migration mode, which is relatively slow. Instead, if Saturn grew more gradually than Jupiter and spent more time near a Saturn-mass, it would have undergone fast migration for a longer period and hence could have trapped Jupiter in resonance. It is unclear why Saturn should have grown more slowly than Jupiter. Possibly, the opacity of the disk increased from the time of accretion of Jupiter to that of Saturn, thus slowing down the gas-accretion rate onto the planet.

Interestingly, among the collection of extra-solar planets, we see at least three systems where the Saturn-analog did not capture in resonance the Jupiter-analog (HD 12661, HD 13498, HIP 14810). But in these cases, the inner, more massive planet is closer than 1 AU to the star and the outer, lighter planet is more than three times further away. This is obviously very different from the orbital architecture of Jupiter and Saturn, or of the system OGLE-06-109L (which is a sort of twin of the Jupiter-Saturn system), which suggests that a different evolution occurred in these cases. I argue that the capture in resonance between a Jupiter-analog and a Saturn-analog is an event that may or may not happen, according to the accretion histories of these planets; depending on this binary possibility, the systems evolve along clearly different paths.

Let's admit now that Jupiter and Saturn got captured in their mutual 2/3 resonance. Morbidelli and Crida (2007) showed that the subsequent dynamical evolution of these planets depends on the properties of the disk, particularly the scale height. For thick disks (about $6 \%$ in scale height for a typical viscosity), the migration is very slow, and the planets remain at effectively constant distance from the central star. But for disks with decreasing thickness, outward migration becomes increasingly fast. In principle, in thin disks outward migration can bring the planets up to ten times further than their initial location in a few thousands orbital periods (Crida et al., 2009), which might explain the orbits of some of the planets discovered by direct imaging beyond several tens of AUs from their parent stars. Until now, outward migration of Jupiter and Saturn from inside $\sim 4 \mathrm{AU}$ was considered incompatible with the existence of the asteroid belt - therefore only proto-Solar disk models which prohibited outward migration were considered viable (Morbidelli et al., 2007). However, as shown by Walsh et al. (2011) and discussed in Sect. 4.1, this is not true, releasing the constraints against Jupiter's outward migration. This opens a new degree of freedom to model the evolution of the solar system, as it will be shown at the end of this chapter.

For two giant planets to avoid inward migration as discussed above, it is essential that the mass of the outer planet is a fraction of the mass of the inner planet, as in the Jupiter-Saturn 
case (Masset and Snellgrove, 2001; Morbidelli and Crida, 2007). I provide here an heuristic, but intuitive explanation of this statement. When two giant planets are close enough to each other, they evolve inside a common gap of the gas-density distribution. The inner planet, being closer to the inner edge than to the outer edge of the common gap, feels a positive torque and would tend to migrate outwards; the outer planet, being closer to the outer edge of the common gap, feels a negative torque and would tend to migrate inwards. If the planets are locked in resonance their relative orbital separation cannot change (if they are not yet in resonance they move towards each other until they are captured and locked into a resonance). Thus, the direction of migration of the pair of planets depends on which of the two torques dominates. Each torque is proportional to the surface density of the disk adjacent to the planet and to the square of the mass of the planet itself (Goldreich and Tremaine, 1979). Because the planets deplete partially the disk in the region between the star and their innermost orbit (a partial cavity; Crida and Morbidelli, 2007), the surface density in the outer disk is typically larger than in the inner disk; so, a necessary condition to avoid inward migration (i.e. to make the torque felt by the inner planet larger than that felt by the outer planet) is that the inner planet is more massive.

Therefore, this mechanism predicts that no pair of resonant giant planets with narrow orbital separation, with the outer planet significantly lighter than the inner one, should ever be found close to the parent star. In fact, planets in this configuration should have avoided inward migration. So far, this prediction is validated by observation. I fact, there are many pairs of planets in resonance (or close to), near their star, but none of these cases exhibits a Jupiter/Saturn mass ratio. The absence of this configuration, which is statistically significant if one assumes that the mass ratio should be random, strongly supports the theoretical result that resonant planets in Jupiter/Saturn mass ratio move outwards, and therefore cannot be found within the range of stellar distances that can be probed by radial velocity observations (OGLE-06-109L system was in fact discovered by micro-lensing).

There is an intriguing aspect in this view of the evolution of Jupiter and Saturn. When the two planets do not migrate inwards at the nominal Type II migration rate, there is necessarily an inward flow of gas, from the outer disk to the inner disk, through the common gap. Thus, the outer planet should presumably accrete more of the incoming material, narrowing the mass difference with the inner planet. This raises the question of why Saturn remained smaller than Jupiter. The answer may be that the gas disk was rapidly disappearing while Jupiter and Saturn were undergoing the dynamical evolution described above, so that Saturn failed to grow further. There are several lines of evidence in favor of a formation of Jupiter and Saturn in a dissipating disk. First, Jupiter's atmosphere is enriched in elements heavier than helium by a factor of 3-4 relative to solar composition (Wong et al., 2004), while Saturn is enriched by a factor 11 in Carbon (Fouchet et al., 2009). Guillot and Hueso (2006) have argued that the easiest explanation for this fact is that hydrogen and helium had been already depleted by a factor of 3-4 in the disk by the time Jupiter captured its atmosphere (and, following this logic, by a factor of 11 by the time Saturn captured its atmosphere). Second, the favored model for the accretion of the regular satellites of 
the giant planets also requires that said satellites formed in gas-poor circum-planetary disks (Canup and Ward, 2006). Third, the common explanation for why Uranus and Neptune failed to accrete massive atmospheres is that the gas disappeared before they had a chance to do so (Pollack et al., 1996). All these arguments suggest that the 4 giant planets formed in a temporal sequence, from Jupiter to Saturn and then to Uranus and Neptune, while the disk was being dispersed. Finally, recall that the Solar composition is at the low-end of the metallicity range for planet-bearing stars. In the core-accretion model of giant planets, the metallicity of the star is correlated with the speed of accretion of the cores, so that stars that are too poor in metals fail to form giant planets before the disappearance of the disk (Ida and Lin, 2004). This suggests that the solar system barely made its giant planets, while the disk was being dispersed.

If this explanation may be satisfying for our solar system, it is nevertheless interesting to discuss what would have happened if Jupiter and Saturn had formed earlier, when the disk was still massive. The issue is not only academic, as it can be pertinent for systems with a higher initial metal content which, as suggested above, should form planets faster. In a gas rich disk, Saturn would have eventually become as massive, or even more massive, than Jupiter. Thus, the two planets would have resumed an inward migration (Morbidelli and Crida, 2007). The orbital eccentricity of two planets migrating in resonance tends to increase monotonically (Ferraz-Mello et al., 2003; Kley et al., 2004). This effect is contrasted by the action of the disk, which tends to damp the planets' eccentricities (Kley and Dirksen, 2006). A crucial role is played by the disk inside the orbit of the inner planet. This inner disk tends to be partially depleted due to the presence of the planet(s). The level of depletion depends on several parameters such as the viscosity of the disk, its scale height, its inner radius, the mass of the planet(s) relative to the disk etc. (Crida and Morbidelli, 2007). If the inner disk is depleted substantially, the damping effect on the eccentricity of the inner planet is strongly reduced. In this situation, the eccentricity of the inner planet keeps growing, until the pair of planets becomes dynamically unstable and mutual close encounters are triggered (Kley et al., 2005). This may be the case of many, if not most, of the planetary systems. In fact, mutual scattering seems to be the major mechanism responsible for the eccentricity distribution observed in the extra-solar planets collection (see sect. 2.3 for a more complete discussion). However, if the inner disk is not very depleted, the eccentricities of the planets grow until a limit value is achieved (Crida et al., 2008; see Fig. 2). This process can leave the planets at the disappearance of the disk on stable resonant orbits with moderate eccentricities and thus it can explain the pairs of planets in resonance observed to date.

In conclusion, we have seen in this section the first crucial "bifurcations" in planetary evolution that can account for at least part of the great diversity observed in planetary systems. In fact, assuming that giant planets form in sequence at increasing distances from the central star, most of the observed diversity of planetary systems could stem from the occurrence or avoidance of two events: (i) the capture in resonance of the first, inner planet by the second, initially smaller one, which stops inward migration (often triggering outward migration) and (ii) the growth of the outer planet beyond the mass of the inner one, which causes inward migration of both planets to resume. 


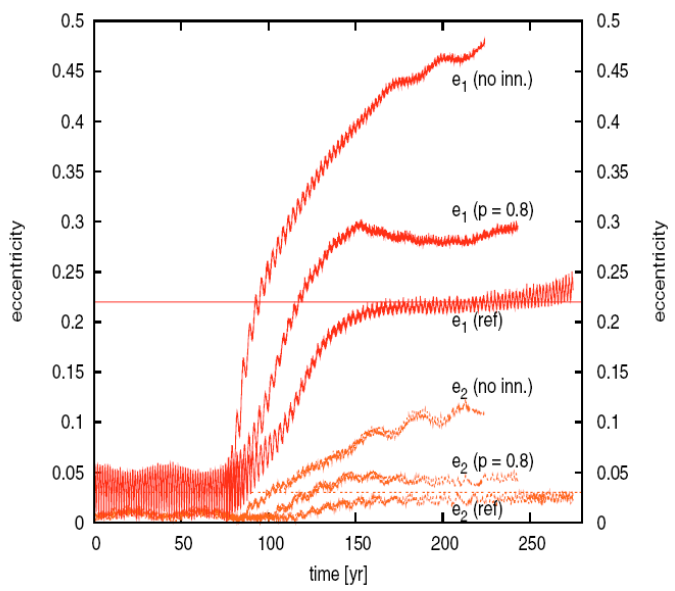

Fig. 2. - The evolution of the eccentricities of planets $b$ and $c$ around GJ876 during their putative inward resonant migration. The eccentricity of the interior planet is labelled $e_{1}$, that of the exterior planet $e_{2}$. The evolutions labelled "no inn." assume that no disk is present inside the orbit of the interior planet; in this case the eccentricities seem to grow indefinitely. The evolutions labelled "ref." and " $\mathrm{p}=0.8$ " account for an inner disk, and differ just for the value of a technical simulation parameter. In these cases the eccentricities attain equilibrium values. In the "ref" case, the final eccentricities reproduce the eccentricities inferred from observations (horizontal lines). From Crida et al., 2008.

The Solar System structure results from the occurrence of (i) and avoidance of (ii). Systems like HD 12661, with a close-in massive planet and a distant smaller planet, result from the avoidance of (i). Resonant giant planets close to their stars, like those in the GJ876 system, result from the occurrence of both (i) and (ii). Unstable systems, ultimately leaving behind one giant planet on an eccentric orbit, may also result from the occurrence of both (i) and (ii), but in cases where there was not enough eccentricity damping because of a depleted inner disk.

Here, the case with two planets was the only one considered; obviously, the tree of possible evolutions can only become more complicated if more giant planets are involved, and the final outcomes can be even more diverse.

\subsection{Planet-planet scattering as the dominant orbital excitation process}

One of the greatest surprises that came with the discovery of extra-solar planets is the realization that most planets have orbital eccentricities much larger than those characterizing the planets of our solar system. Eccentricities of about 0.4 are quite common in the extra-solar planets collection; some planets have eccentricities larger than 0.6 , values that in our solar system are common only for comets!

Whatever the preferred model of giant planet formation (core-accretion or gravitational instability; see sect. 2.1), it is expected that planets have originally small orbital eccentricities, because 


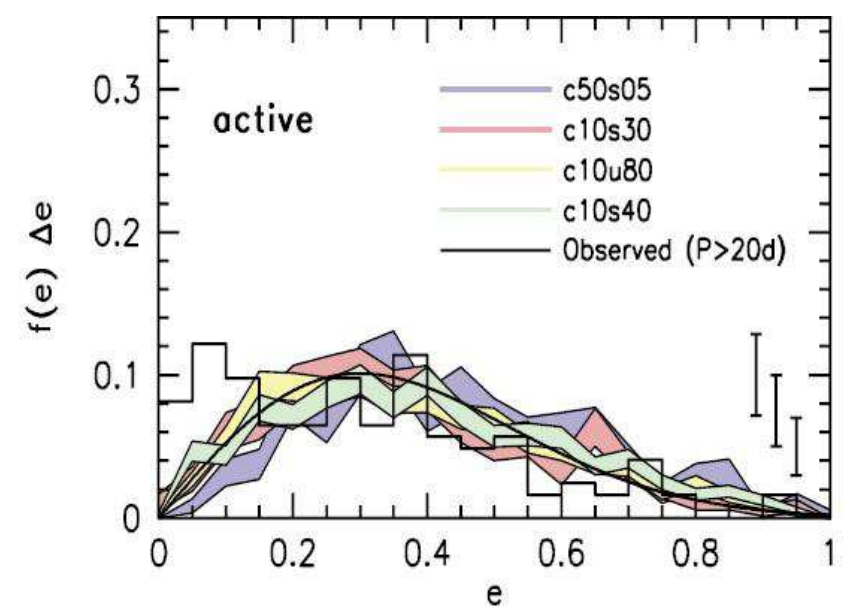

Fig. 3.- Final eccentricity distribution of simulated ensembles of planetary systems that underwent a dynamical instability sometime during the full simulation time-span of $10^{8} \mathrm{y}$. The color bands correspond to different ensembles, characterized by different initial conditions. The histogram shows the observed eccentricity distribution of extra-solar planets with orbital period longer than $20 \mathrm{~d}$, according to Butler et al. (2006). The observed distribution and the final distributions resulting from the simulations agree very well, with the exception of an excess of observed planets with $e<0.2$. This is probably due to planets that never underwent a significant dynamical instability. From Juric and Tremaine (2008).

they form from a circum-stellar disk whose streamlines are basically circular. There has been a lively debate on whether subsequent planet-disk interactions can raise the planets' eccentricities up to the observed values. Papaloizou et al., (2001) concluded, with numerical experiments and theoretical considerations, that eccentricity growth is not possible for planetary masses below 10-20 Jupiter masses. Instead, Goldreich and Sari (2003) argued with theoretical considerations that, under some conditions, depending mostly on disk's thickness, giant planets of more moderate masses could have their orbital eccentricity excited, although they could not estimate the magnitude of this excitation. More recent hydro-dynamical simulations (D'Angelo et al., 2006; Kley and Dirksen, 2006) showed that planets with masses larger than $\sim 2-3$ Jupiter masses, under some conditions, can have eccentricities excited by the disk, but only to moderate values $(\sim 0.1-0.2)$, definitely lower than those characterizing many, if not most, of the extra-solar planets. In most cases, the planet-disks interactions rather seem to lead to eccentricity damping. Thus, a more generic orbital excitation mechanism seems to be required to explain the observations.

Soon after the discovery of the first eccentric planets, it was pointed out that mutual encounters between planets can easily provide strong orbital excitation (Rasio and Ford, 1996; Weidenschilling and Marzari, 1996; Lin and Ida, 1997; Levison et al., 1998; Ford et al., 2001; Marzari and Weidenschilling, 2002; Adams and Laughlin, 2003). More recent studies (Juric and Tremaine, 2008; Chatterjee et al., 2008; Ford and Rasio, 2008; Raymond et al., 2009) show that random systems of giant planets initially on unstable, quasi-circular orbits evolve through close encounters until a dynamical relaxation state is achieved with the ejection or collision of some planets. In these models the final eccentricity distribution of the surviving planets is remarkably similar to that of 


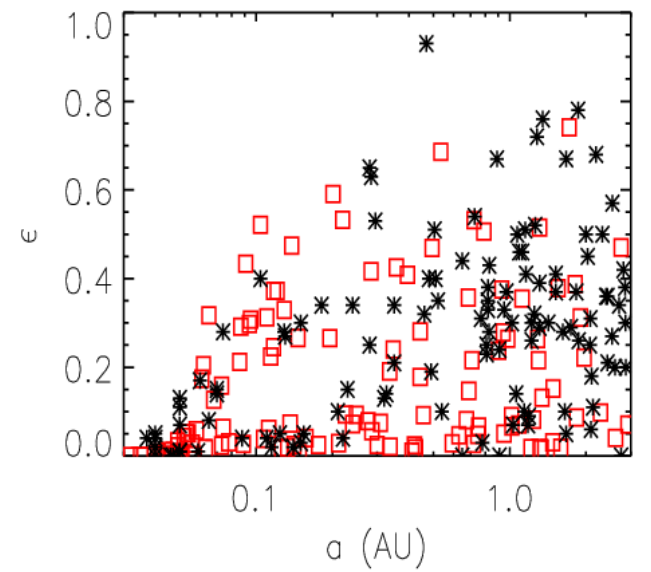

Fig. 4.- The final semi major axis vs. eccentricity distribution of extra-solar planets (squares) in the simulations of Moorhead and Adams (2005) which account for (i) inward migration, (ii) eccentricity damping due to the disk (with an assumed timescale of $0.3 \mathrm{My}$ ) (iii) tidal circularization and (iv) radial velocity detection biases. The stars show the distribution of the extra-solar planets known at the time.

known extra-solar planets (see Fig. 3). Moreover, Juric and Tremaine (2008) and Raymond et al. (2009) showed that the final orbital spacing of the surviving planets is also in good agreement with the observations of extra-solar systems of two or more non-resonant planets. These systems typically look "packed", in the sense that the orbital separation (apocenter to pericenter) between neighboring planets is not much larger than what is required by the Hill-stability criterion (Barnes and Greenberg, 2006). All these quantitative results give strong support to the idea that planetplanet scattering is the main mechanism sculpting the orbital distribution of extra-solar planets. In addition, Veras et al. (2009) pointed out that planet-planet scattering can propel a planet in the region beyond $100 \mathrm{AU}$, which can explain some of the extra-solar planets imaged at large distances from their parent stars? 2 .

It is important to remember that, assuming giant planets formed beyond an hypothetical snowline at 3-5 AU, the planet-planet scattering mechanism alone cannot explain the observed semi major axis distribution of extra-solar planets (see for instance Marzari and Weidenschilling, 2002). In fact, through scattering events, planets can have difficulty reaching orbits with semi major axis smaller than half of the initial value of the inner planet, with the exception of those objects scattered onto orbits with very large eccentricities and small periastron distances, which may then become circularized by tidal dissipation. Thus, migration is still required to explain

\footnotetext{
${ }^{2}$ In summary, three mechanisms have been proposed to move planets from the snowline region to large distances from the central star: (i) outward Type-II migration of planets originally formed in the outer part of a disk in rapid viscous spreading (Veras and Armitage, 2004); (ii) outward migration of a pair of resonant planets with a JupiterSaturn mass hierarchy (Crida et al., 2009); (iii) scattering of a planet to a wide elliptic orbit (Veras et al., 2009). These mechanisms are potential alternatives to the possibility that distant planets formed in-situ, by the gravitational instability mechanism (Boley et al., 2009)
} 
the presence of a large number of planets on orbits with small semi major axes. Adams and Laughlin (2003) and Moorhead and Adams (2005) modeled the interplay between migration and scattering. They used N-body simulations, with fictitious forces to mimic the effect of the disk on the planets, for what concerns both semi major axis decay and eccentricity damping. After tuning a few parameters (the lifetime of the disk, the timescale of the eccentricity damping, etc.) and accounting for observational biases, Moorhead and Adams obtained a very good reproduction of the two-dimensional $(a, e)$ distribution of the exoplanets detected by the radial velocity technique (Fig. (4). Although very appealing, this result may be questioned because the numerical recipes used to mimic migration and damping have been based on simple analytic estimates. Reality may be more complicated. For instance, as we have seen above, a system of two planets in resonance may migrate at a very different speed than a single planet in Type II migration (Morbidelli and Crida, 2007); the disk may not always damp the eccentricity of a planet, but could also sustain it, depending on the planet mass and initial eccentricity (Kley and Dirksen, 2006); migration direction could be reversed for eccentric planets (D'angelo et al., 2006); finally mass accretion onto the planets is neglected in the Moorhead and Adams model.

For all these reasons, I think that two key questions have not yet been answered in a definitive way: why do giant planets become unstable in the first place? And when do they become unstable, relative to the gas disk lifetime? I speculate a bit on both issues below.

Concerning the first question, there are in principle two answers: either planetary systems become unstable because the planets grow in mass, or because the planets are brought too close to each other by migration processes. I would tend to exclude the first case as a dominant mechanism to explain the large eccentricities of extra-solar giant planets, for the following reason. Imagine a system of cores close to each other (maybe brought by Type I migration into mutual resonances of type $n /(n+1)$, with quite large $n)$. Given that the time required to trigger the runaway accretion of a massive atmosphere is much longer than the accretion of the atmosphere itself (Pollak et al., 1996), it is unlikely that all the cores would accrete massive atmospheres and become giant planets simultaneously. More realistically one core would start the accretion of the atmosphere first, and it would acquire a mass much larger than those of the other planets at that time. The system would become unstable, because it is too closely packed to stand the newly born giant planet. However, the scattering phase would bring the cores onto orbits with large eccentricities but it would leave the newly born giant planet onto a quasi-circular orbit: this does not correspond to the orbital distribution of the extra-solar planets that we see. Moreover, the orbital eccentricities of the cores would be damped very fast by the disk (Cresswell et al., 2007; Bitsch and Kley, 2010) and, by the time these cores become in turn giant planets, they would be again on quasi-circular orbits. I'm not denying that this kind of evolution can occur. A system of multiple giant planets probably forms through several repetitions of the events described above 3 . My claim is that this is probably

\footnotetext{
${ }^{3}$ The reader should remember that two planets are stable if their orbital separation is a few times their mutual Hill radius $R_{H}=\bar{a}\left[\left(m_{1}+m_{2}\right) / M_{S}\right]^{1 / 3}$, where $m_{1}$ and $m_{2}$ are the masses of the two planets and $\bar{a}$ is their mean
} 


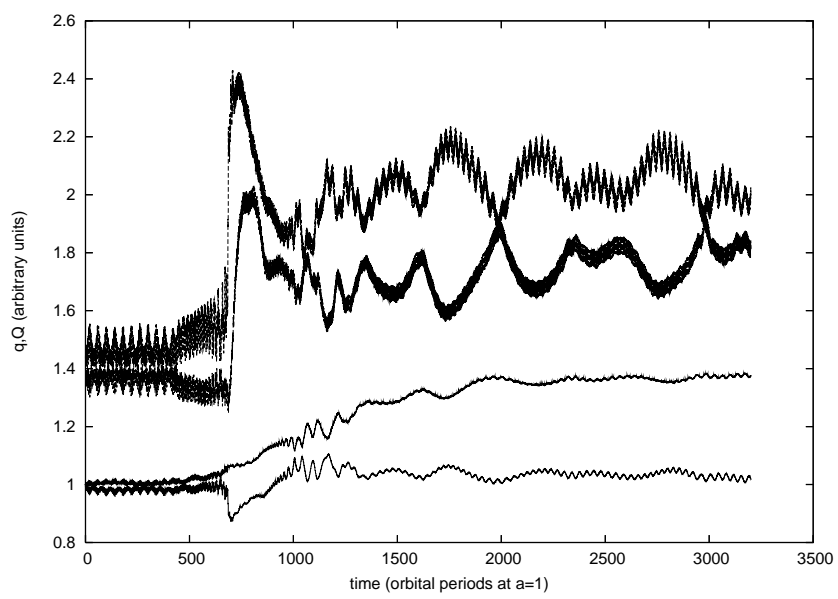

Fig. 5. - The evolution of a pair of giant planets initially on unstable orbits. For each planet, the pair of curves shows the evolution of the periastron $(q)$ and apoastron $(Q)$ distances. Thus, when the two curves are close, the orbit is almost circular and $q \sim Q \sim a$, where $a$ is the orbital semi major axis. The light curves at the bottom are for the inner planet, with a 3 Jupiter mass; the thick curves at the top are for the outer planet, with one Jupiter mass. Notice that the orbits become very eccentric and separate from each other at the time of the instability, after about 500-700 orbits of the inner planet. Subsequently, the eccentricities of the planets are damped and the inward migration of the outer planet brings it in the $1 / 2$ resonance with the inner one $(t=1,000)$. Once in resonance, the eccentricity increases again and starts to have long-period oscillations. A relatively stable configuration is achieved. From Morbidelli and Crida (2007).

not the process that sculpts the final orbital distribution of giant planet systems. I think that a more generic mechanism to achieve the ultimate dynamical instability (the one responsible for the final orbits) is that two or more giant planets, once fully formed, are brought in resonance with each other by Type II migration, which causes a subsequent increase of their orbital eccentricities (Ferraz-Mello et al., 2003; Kley et al., 2004, 2005; Crida et al., 2008).

This brings us to the second question, on the timing of the instability. In the Adams and Laughlin (2003) and Moorhead and Adams (2005) models, the planets become unstable while they are still migrating in the gas disk. However, our limited experience with hydro-dynamical simulations of giant planets scattering each other in gas-disks shows that the evolution can be quite different from the one modeled in those works. Scattered planets tend to acquire orbits which are more separated in semi major axis and more eccentric, but then the eccentricities are damped by the disk and the planets migrate back into a new, more stable resonant configuration, with moderate

semi major axis, while $M_{S}$ is the mass of the star. Suppose now that planets tend to acquire orbits whose mutual separation is not much larger than this Hill-stability limit. If a planetary system is made of two planetary cores, when one of two objects becomes a giant planet, the system is likely to be destabilized because $R_{H}$ increases by a factor 3-4 as the mass of one planet grows by a factor 30-60. Instead, a stable system made of one giant planet and one core is not likely to be strongly destabilized when the core grows to the status of a giant planet, because $R_{H}$ increases only by a factor $\sim 2^{(1 / 3)}=1.25$. Similarly, in a system made of one giant planet and two cores, the growth of one of the cores to the status of a giant planet is likely to destabilize the remaining core, but not the first planet. 
eccentricities (see for instance Fig. 5 or Moekel et al., 2008). It is possible that systems with more planets develop more violent instabilities that extend also longer in time. However, new simulations with three planets, presented in Marzari et al. (2010), again show that the planets surviving at the end of the instability phase have low-eccentricity orbits. Until we know more from hydro-dynamical simulations about the dynamics of eccentric planets, it is premature to conclude whether instabilities produced during the gas-disk phase would lead to the observed orbital distribution of extra-solar planets. The other possibility is that during the gas disk phase planets acquire stable resonant and eccentric orbital configurations, which become unstable when the gas is removed. This is the approach of Lin and Ida (1997), Levison et al. (1998), Juric and Tremaine (2008), Chatterjee et al. (2008), to quote just a few works.

The facts that most extra-solar planets have quite large eccentricities and that there are only a few pairs of stable resonant planets, suggest that orbital instability in planetary systems is more the rule than the exception. I think that it would be quite surprising if most planet configurations achieved through migration were stable in presence of gas and unstable in absence of gas. In fact, the gas has some stabilizing effect due to the eccentricity damping that it exerts, but it also drives migration, which in turn excites the eccentricity. The two effects cancel out when a limit eccentricity is attained (Fig. 2). At this point, the gas should not play any longer any crucial role in maintaining stability. One possibility is that most resonant configurations achieved through migration are unstable in both cases (with gas and without gas), but the instability manifests itself on timescales of several millions of years, i.e. well after that the gas has been removed. To have a better appreciation of reality, we need more systematic hydro-dynamical simulations of the dynamics of sets of giant planets embedded in gas-disks, followed by the investigation of their subsequent long-term evolutions after the gas removal. A work of this kind has been done for the planets of our solar system (Morbidelli et al., 2007; see sect 2.4), but it is obviously more demanding in general, given the volume of parameter space that needs to be explored.

Naively, I would have expected that planets that develop instabilities and mutual encounters after having migrated to the vicinity of the central star acquire smaller eccentricities than those that do so further away from the star. This is because the eccentricity acquired in an encounter is proportional to the ratio between the velocity kick received during said encounter and the orbital velocity. The former depends on the escape velocities from the surface of the planets and is independent of the orbital radius, while the latter is larger for the close-in planets. Instead, Juric and Tremaine (2008) showed numerically that the eccentricity distribution achieved at dynamical relaxation (i.e. after many encounters) is essentially independent of the semi major axes of the planets. Given that we do not see any clear correlation between distance and eccentricity in the extra-solar planets collection (apart from the tidal circularization zone) this result gives a quite strong support to the idea that planets first migrate towards the central star and then, after gas removal, become unstable. 

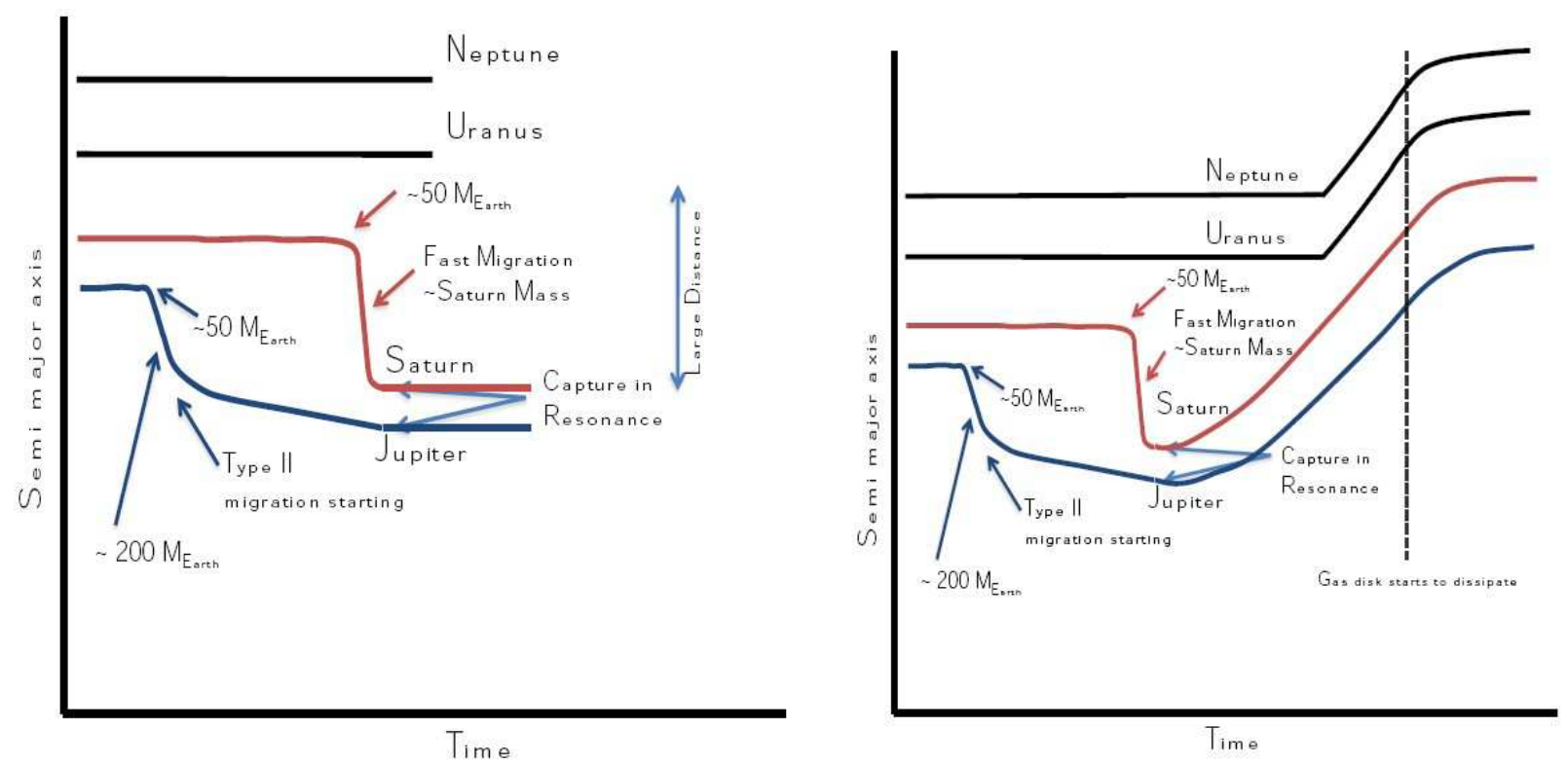

Fig. 6. - Sketch of the possible migration and accretion histories of the giant planets of the solar system, while they were embedded in the disk of gas. Both scenarios assume that: (i) Saturn was eventually caught Jupiter in their mutual 2/3 resonance (see sect. 2.2 for conditions) and (ii) planets with masses smaller than $\sim 50$ Earth masses do not migrate in radiative disks. In the left panel, Jupiter and Saturn do not migrate after resonance capture. This leaves a large gap between the Jupiter/Saturn pair and the Uranus/Neptune pair, that cannot be reconciled (to our current knowledge) with the current orbital structure of the planets. In the right panel, Jupiter and Saturn migrate outwards. This leads to the capture of Uranus and Neptune in resonance with Saturn and with themselves. This resonant configuration is consistent with the current orbits of the planet via a phase of dynamical instability after the dispersal of the disk of gas (see sect. 3.2).

\subsection{A plausible evolution of the four giant planets of the solar system}

I now come back to our solar system, to outline a plausible scenario for the evolution of the 4 major planets during the gas-disk phase. As we have seen above (sect. 2.2), hydro-dynamical simulations strongly suggest that Jupiter and Saturn rapidly reached a $2 / 3$ resonant orbital configuration and that this prevented them from migrating further towards the Sun. Once in resonance, these giant planets either remained on non-migrating orbits or migrated outwards.

What about Uranus and Neptune? According to the latest models on migration of planetary cores in radiative disks, Uranus and Neptune, or their precursors, should have evolved on nonmigrating orbits in the intermediate part of the disk (Lyra et al., 2010). If Uranus and Neptune did not migrate while Jupiter and Saturn achieved non-migrating orbits after a period of inward migration, eventually the giant planets system should have been characterized by a large separation between the Jupiter/Saturn pair and the Uranus/Neptune pair (see left panel of Fig. 6). How to reconcile this peculiar orbital structure with the current structure of the solar system is unknown. Instead, if Jupiter and Saturn migrated outwards, Uranus and Neptune would have been caught in 
mean motion resonances with the two major planets (see right panel of Fig. 6). The same would have occurred also in the case where Jupiter and Saturn remained on non-migrating orbits but Uranus and Neptune started to migrate inwards due to a change of the thermal properties of the disk over time (Lyra et al., 2010). What matters, in fact, is the convergent migration between the Jupiter/Saturn pair and the Uranus/Neptune pair, not which pair of planets is moving.

A search for possible resonant configurations that could have been achieved by the two pairs of planets in convergent migration has been done in Morbidelli et al. (2007), using hydro-dynamical simulations, and in Batygin and Brown (2010), using N-body simulations with fictitious forces that mimic the effect of the disk. The search is probably not complete, in the sense that other relative resonant configurations might have been achieved, depending on disk properties, migration speed and initial Uranus/Neptune configuration. Three results, however, seem robust. First, in case of convergent migration, each planet ends up in resonance with its neighbor. Thus, the planets should have been in a 4-body-resonance, the most complex multi-resonant configuration in the solar system 4 . Second, because distant resonances are weak and have a low probability to capture a migrating body, the four planets most likely should have achieved a very compact orbital configuration. Third, the eccentricities of the planets should have remained small (less than 0.005 for Jupiter, 0.02 for Saturn, 0.06 for Uranus and 0.015 for Neptune in Morbidelli et al. simulations). Thus Jupiter and Saturn should have been on orbits significantly more circular than now.

Morbidelli et al. also investigated the long-term stability of resonant orbital configurations that they found. This was done by continuing each hydro-dynamical simulation for 1,500 Jupiter's orbits, while removing uniformly the gas, exponentially in time, down to a factor of $1 / 1,000$. This procedure was just instrumental for changing adiabatically the potential felt by the planets, and was not intended to mimic the real process of evaporation of the disk. The final orbits of the planets were then passed to a symplectic N-body code, and integrated for $1 \mathrm{~Gy}$, without additional perturbations (for instance from a planetesimal disk). Morbidelli et al. found that only one configuration was stable for $1 \mathrm{~Gy}$. This result was actually affected by an error of the integrator, which was discovered only later. In reality, 4 of the 6 configurations found in Morbidelli et al. are stable for $1 \mathrm{~Gy}$. The only unstable configurations are the two most compact ones, with Uranus in the $3 / 4$ resonance with Saturn and Neptune in either the $4 / 5$ or $5 / 6$ resonances with Uranus. These configurations lead to very violent instabilities, with close encounters of all the planets with each other, including close encounters of Jupiter with Saturn. In these cases, orbital relaxation is achieved when all planets except Jupiter are ejected on hyperbolic or distant eccentric orbits. Obviously this is not what happened in our solar system. But, in these simulations, the final orbit of Jupiter, with an eccentricity of 0.4 , is similar to those of the extra-solar planets discovered to date at a distance of 4-5 AU from their parent stars. This suggests that these extra-solar planets might be the survivors of systems that avoided Type II migration, possibly through a mechanism like the Jupiter-Saturn

\footnotetext{
${ }^{4}$ Currently the record for the most complex resonance chain is detained by Jupiter's satellites Io, Europa and Ganymede, which are locked in a 3-body-resonance, also known as the Laplace resonance.
} 
one, but which achieved orbital resonant configurations so compact to undergo a violent instability involving encounters between gas-giant planets. Our solar system was more lucky, and picked up a less compact multi-resonant configuration, so that encounters between Jupiter and Saturn could be avoided.

We will see in the next section that the least compact resonant configurations, which are stable when only the 4 planets (Jupiter to Neptune) are considered, can become unstable when the interactions of the planets with a remnant planetesimal disk are taken into account. I will argue that the solar system must have passed through such an instability phase, to reconcile the current orbits with those that the planets should have had when they emerged from the gas-disk phase (see also Thommes et al., 1999).

\section{The planetesimal-disk era}

\subsection{Brief tutorial of planetesimal-driven migration}

A planet embedded in a planetesimal disk has repeated close encounters with the objects that come close to its orbit. Each of these encounters modifies the trajectory of the incoming planetesimal and, consequently, the planet has to suffer a small recoil. In this way, if the planetesimal disk is sufficiently massive, significant angular momentum exchange may occur between the planet and the planetesimals, enough to cause a rapid, long-range migration of the planet (Ida et al., 2000). A review of planetesimal-driven migration has been presented in Levison et al. (2007). In this section, I discuss the basic concepts that are relevant to understand the evolution of our solar system and, possibly, of planetary systems in general.

For a system of giant planets, planetesimal-driven migration is relevant only after the disappearance of the gas. The reason is that the gas contains typically $\sim 100$ times more mass than the planetesimals and therefore it exerts the dominant gravitational forces on the planets. Consequently, planetesimal-driven migration was not mentioned in the previous part of this chapter. It should be remembered, though, that for small planets gas-driven migration is proportional to the planet's mass (Ward, 1997), whereas planetesimal-driven migration is, at first order, independent of the planet's mass (Ida et al., 2000; Kirsh et al., 2009); thus, for small planets, such as planetary embryos of an Earth-mass or smaller, planetesimal-driven migration may rival, under some conditions, Type-I migration (Levison et al., 2010; Capobianco et al., 2011).

One may naively think that planetesimals scattering is a purely random process, which consequently cannot force a planet to migrate in a specific direction. This is not true. To zeroth order, during an encounter the planetesimal is in a Keplerian orbit about the planet. Since the energy of this orbit must be conserved, all the encounter can do is to rotate the relative velocity vector. Thus, the consequences of such an encounter can be effectively computed in most of the cases using an impulse approximation (Opik, 1976; Ida et al., 2000). With this approach, it is easy 
to compute that on average (that is averaged on all impact parameters and relative orientations) the planetesimals that cause a planet to move outward are those whose $z$-component of the specific angular momentum $H=\sqrt{a\left(1-e^{2}\right)} \cos i$ is larger than that of the planet $\left(H_{p}\right)$. In fact, during the encounter these planetesimals have an azimuthal angular velocity faster than that of the planet: thus, they tend to be slowed down, propelling in turn the planet along its orbit. The opposite is true for the planetesimals with $H<H_{p}$ (Valsecchi and Manara, 1997). In these formulæ $a, e$ and $i$ are the semi major axis, eccentricity and inclination of the planetesimal.

The direction of migration for a single planet in principle depends on the angular momentum distribution of objects on planet-encountering orbits. Nevertheless, the direction of migration does not simply depend on the sign of $\bar{H}-H_{p}$, where $\bar{H}$ denotes the mass-weighted value of $H$ of the planet-crossing particles: there is a bias in scattering timescales on either side of the planet's orbit which leads to a very strong tendency for the planet to migrate inwards (Kirsh et al., 2009). Consequently, outward migration is found only in systems where $\bar{H}-H_{p}$ is strongly positive, such as for planetesimal disks with surface density distribution proportional to $r^{k}$ with $k>1$ (the value of $k$ for the real disks is expected to be between -2 and -1 , definitely giving inward migration).

In order to understand the basic modes of migration, imagine defining a function $\mathcal{F}$ of the $H$-distribution of the planet-crossing particles, such that the planet's migration rate $\mathrm{d} a_{p} / d t$ is proportional to $\mathcal{F}$ (which implies that migration is inwards if $\mathcal{F}$ is negative, while it is outwards otherwise). For a given orbital distribution of the planetesimals in the planet-encountering zone (i.e. for a fixed value of $\mathcal{F}$ ), the rate of migration in the local units of length and time must be proportional to the total mass $M$ of the planet-encountering planetesimals (Ida et al., 2000; Gomes et al., 2004; Levison et al., 2007). Thus, we can write

$$
\frac{\mathrm{d} a_{p} / a_{p}}{\mathrm{~d} t / T} \sim M \mathcal{F}
$$

where $T$ is the planet's orbital period. When a planet migrates, several concomitant processes occur. (a) The planetesimals that are scattered in the direction opposite to that of planet migration (e.g. onto an orbit with a larger semi major axis, for an inward migrating planet) can be "left behind", in the sense that they can find themselves on orbits which do not cross the planet's orbit any more (because the orbit of the planet has drifted away; see Fig. 7). Conversely, other planetesimals, originally situated on stable orbits in the disk through which the planet is moving, start to be scattered. Thus, planetesimals both enter and leave the planet-encountering region as a result of the radial drift of the planet's orbit through the disk. This orbital drift tends to leave $\mathcal{F}$ unchanged, but changes $M$; the latter increases or decreases depending on the gradient of the disk's surface density. (b) The planet encounters tend to re-arrange the angular momentum distribution of the planetesimals to an equilibrium configuration that would induce no-migration; this decreases $|\mathcal{F}|$ towards zero, while preserving $M$. (c) Some planetesimals may be eliminated from the system through collisions with the planet or ejections onto hyperbolic orbit, which decreases $M$. Depending on whether, as a net result of all these processes, $|M \mathcal{F}|$ increases or decreases, radial migration accelerates exponentially or decays to zero. The former case is called forced or 


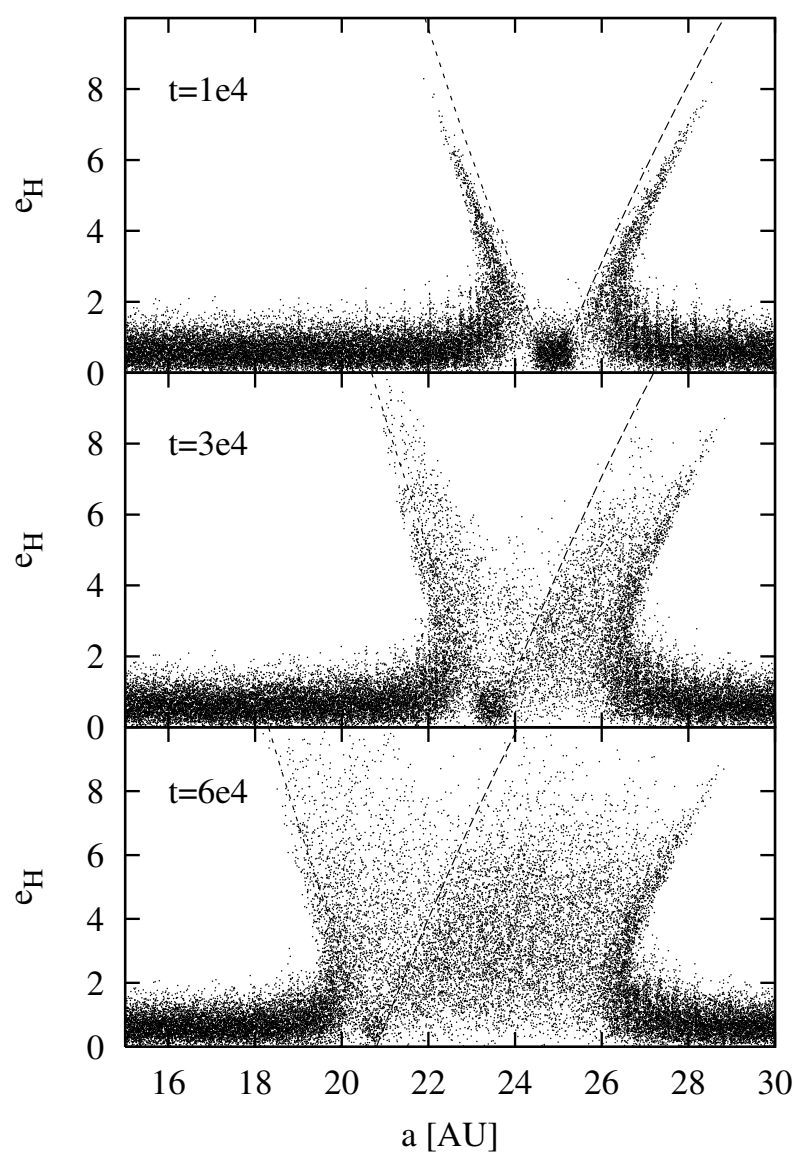

Fig. 7.- The migration of a $2.3 M_{\oplus}$ planet in a planetesimal disk of $230 M_{\oplus}$. Each panel shows the eccentricity vs. semi major axis distribution of the planetesimals (dots) at the time marked in the top left corner. The dashed curves delimit the planet crossing region. The planet is situated at the point of intersection of these curves. Notice how planetesimals are "left behind" on eccentric orbits as the planet migrates inwards. Adapted from Kirsh et al. (2009).

self-sustained migration; the latter is called damped migration. Whereas processes (b) and (c) inexorably tend to damp the planet's migration (because they reduce either $|\mathcal{F}|$ or $M$ ), process (a) can sustain the migration if it leads to an increase of $M$. Thus self-sustained migration occurs if two conditions are met: first, process (a) has to give a positive feedback on migration, which translates into a condition on the gradient of the surface density of the disk; second, the mass $M$ has to be large enough so that the timescale of process (a), which is proportional to $1 / M$, is faster than those of (b) and (c), which are independent of $M$.

The dynamical evolution is qualitatively different if there are two planets. In this case, planetesimals can be scattered by one planet onto an orbit that has close encounters with the other planet. This transfer of particles from the "control" of one planet to the other tends to increase the orbital separation between the planets. However, the planets can effectively move away from 
each other only if they are not locked in a mutual mean motion resonance (the orbital response of resonant planets is different and will be discussed in Sect. 3.2). Assuming no resonance locking, under some conditions, this orbital divergence can lead the outer planet to migrate outwards, even in planetesimal disks in which a single planet would normally migrate inwards. In particular, this is the case for a Neptune-mass planet on an orbit exterior to a Jupiter-mass planet. In fact, the planetesimals that the Neptune-mass planet scatters inwards onto orbits crossing that of the Jupiter-mass planet, are rapidly ejected onto hyperbolic orbits by the latter; conversely, the planetesimals that the Neptune-mass planet scatters outwards, remain on orbits crossing that same planet, and have repeated encounters with it: sooner or later, most of them will be eventually scattered inwards and then removed by an encounter with the Jupiter-mass planet. In conclusions, the net work of the Neptune-mass planet is to transfer planetesimals inwards to the control of the Jupiter-mass planet, and consequently the Neptune-mass planet has to move outwards.

The case of the giant planets of our solar system, with Jupiter in the innermost orbit, two Neptune/Uranus-mass planets on the outermost orbits and one intermediate-mass planet (Saturn) in between, is somewhat analog to the simple Jupiter-Neptune system described above (if, again, the planets are not in resonance and are free to migrate relative to each other). With $N$-body simulations, Fernandez and Ip (1984) showed for the first time that Jupiter migrates inwards, while Saturn and, particularly, Uranus and Neptune move outwards. Malhotra (1993, 1995) elaborated on this kind of evolution to explain the observed orbital properties of the Kuiper belt (a population of planetesimals, including Pluto, with semi major axes beyond that of Neptune). The original version of the "Nice model" 5 (Tsiganis et al., 2005; Gomes et al., 2005), that aimed to build a coherent scenario of the late orbital evolution of the outer solar system, was also based on this process of divergent migration of the giant planets. Given our current understanding that the giant planets, at the end of the gas-disk era, had to be in resonance with each other (see previous section), these models are not strictly valid any more, and consequently I won't discuss them further. A new version of the "Nice model", which starts from a multi-resonant orbital configuration of the giant planets, is instead illustrated in sects. 3.2 and 3.3 .

There are nevertheless some important results of general relevance that these studies brought to light. The concepts of self-sustained migration and damped migration apply to the multi-planet case as well. In the case of self-sustained migration, Neptune tends to migrate to the outer edge of the planetesimal disk, or at least up to a large distance ( $100 \mathrm{AU})$ from the Sun (Gomes et al., 2004). In the case of damped migration, the planets attain a final orbital configuration after having removed all planetesimals in between them and leaving a massive disk of objects a few AUs beyond the final orbit of Neptune (Gomes et al., 2004). Given that the Kuiper belt contains very little mass (probably less than $0.01 M_{\oplus}$; Fuentes and Holman, 2008) and excluding, from considerations based on its orbital and size distributions, that said little mass is the result of collisional grinding (Morbidelli et al., 2008b; see however Kenyon et al, 2008 for an opposite view), my conclusion

\footnotetext{
${ }^{5}$ Named for the French city of Nice, where it was developed.
} 
is that the original planetesimal disk in our solar system had to have an effective outer edge at $\sim 30$ AU, close to the current location of Neptune (Gomes et al., 2004; Morbidelli et al., 2008b).

Another general result is that, during planetesimal-driven migration, the eccentricities of the planet are damped. This is related to a process called dynamical friction, well known in models of planet formation. In essence, dynamical friction is the mechanism by which gravitating objects of different masses exchange energy so as to evolve towards an equipartition of energy of relative motion (Saslaw, 1985): as a general rule, for a system of planets embedded in a massive population of small bodies, the eccentricities and inclinations of the former are damped, while those of the latter are excited (Stewart \& Wetherill, 1988). Thus, during planetesimal-driven migration, the only possibility for the enhancement of the planets' eccentricities is that the planets pass through mutual resonances as their orbits diverge from each other (Chiang, 2003; Tsiganis et al., 2005).

\subsection{Multi-resonant planet configurations and planetesimal scattering: the Solar System case}

When two planets are in a mutual mean motion resonance, their orbits cannot move freely relative to each other: the resonance holds constant the ratio between the orbital periods, i.e. between the orbital semi major axes. Thus, both planets have to migrate at the same relative rate. In this case, the differential forces that act on the planets from the planetesimals disk modify instead the orbital eccentricities of the planets. More precisely, if the interaction with the planetesimals is such that, in absence of the mean motion resonance, the planets would suffer divergent migration (i.e. the ratio between the semi major axes of the outer and the inner planets would increase), the eccentricities of the planets decrease (Henrard, 1993). When the eccentricities are low enough, the planets can eventually exit their resonance. At this point, if the planets are still stable, normal divergent migration, as discussed in the previous section, can start.

Let's consider now the case of the solar system. Assume for instance that the planets were in the least compact of the multi-resonant configurations found in Morbidelli et al. (2007): Saturn is in the $2 / 3$ resonance with Jupiter, Uranus is in the $2 / 3$ resonance with Saturn and Neptune is in the $3 / 4$ resonance with Uranus. Assume that the system, at the disappearance of the gas, is still embedded in a planetesimal disk, of about $50 M_{\oplus}$, with an outer edge at $\sim 30$ AU. Fig. 8 shows what happens: after a short time, the planets are extracted from their multiple resonance. Resonances have a strong stabilizing effect for close orbits (a clear example is that of Pluto which, despite it crosses the orbit of Neptune, is stable because it is in the $2 / 3$ resonance with it). Once the planets are extracted from their mutual resonances, this stabilizing effect ends. The planets rapidly become unstable, because they are too close to each other. In the simulation illustrated in Fig. 8 , the onset of the instability occurs at $t \sim 2 \mathrm{My}$. Uranus and Neptune start to have close encounters with each other and with Saturn. As a result, the orbits of all the planets become more eccentric; Uranus and Neptune are propelled outwards by encounters with Saturn, onto very eccentric orbits. Their orbital eccentricities are then strongly damped by dynamical friction, which stabilizes the 


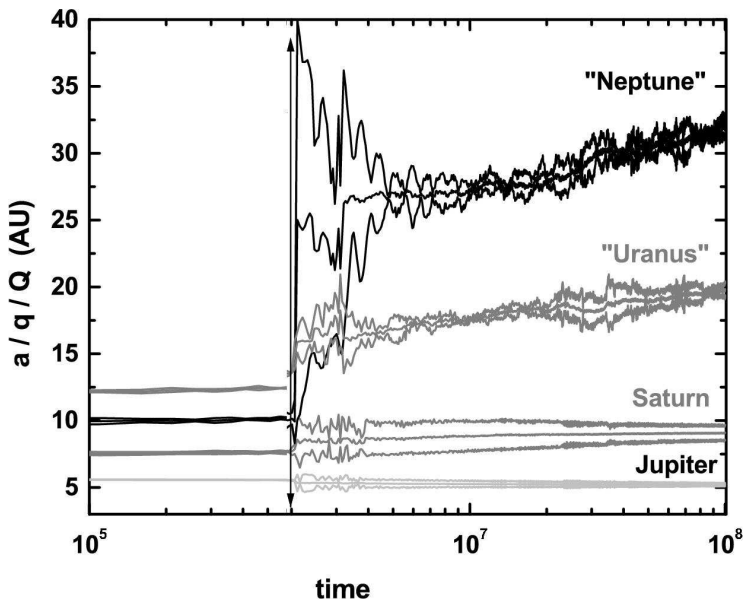

Fig. 8. - The evolution of the 4 giant planets of the solar system, starting from a 4-body resonance and embedded in a $50 M_{\oplus}$ planetesimal disk. Here, each planet is represented by three curves, showing the perihelion distance $q$, the semi major axis $a$ and the aphelion distance $Q$, respectively. Initially Saturn is in the $2 / 3$ resonance with Jupiter, Uranus is in the $2 / 3$ resonance with Saturn and Neptune is in the 3/4 resonance with Uranus. The vertical arrow marks the time of the instability, when the orbits of the planets are extracted from the original 4-body resonance and become eccentric. In this simulation, Uranus and Neptune are scattered outwards by encounters with Saturn and between themselves. The final orbits are quite similar to the current orbits. From Morbidelli et al. (2007).

motion of the planets and prevents further mutual close encounters; the eccentricities of Jupiter and Saturn are also damped, but to a lesser extent. At the same time, planetesimal-driven migration, which can operate once the planets are extracted from resonance, increases further the orbital separations between each pair of planets. At the end of the simulation, the planets have acquired orbits very similar to their current ones: the semi major axes are within $10 \%$ of the real values, and the eccentricities and inclinations are also comparable. Most of the planetesimal disk has been dispersed by then, so that little orbital evolution is expected to occur after $100 \mathrm{My}$.

This simulation shows that the multi-resonant configuration, which the giant planets should have been driven into during the gas-disk phase, is not incompatible with the current orbital configuration: the interaction with the planetesimals disk and the temporary phase of global instability, which the planets experience after extraction from their original resonances, can very well lead the system to its current dynamical state (Thommes et al., 1999). More examples of this kind of successful evolution, starting also from different multi-resonant orbital configurations, can be found in Batygin and Brown (2010). 


\subsection{The Late Heavy Bombardment as a smoking gun for a late instability of the giant planets}

In Fig. 8 the dynamical instability occurs early, after only 2 My from the beginning of the simulation. However, there is a strong indication that in our solar system the onset of the dynamical instability happened much later, approximately 600 My after the disappearance of the disk of gas: this piece of evidence comes from the so-called "Late Heavy Bombardment" (LHB).

The LHB is a cataclysmic period between $\sim 4.0$ and $\sim 3.8$ Gy ago, marked by an extraordinarily high rate of collisions on the Moon (Tera et al., 1974; Ryder, 1990, 2002; Cohen et al., 2000; Ryder et al., 2000). Some authors still contend the existence of such a spike in the history of the bombardment rate (see for instance Baldwin, 2006; Hartmann et al., 2007) and interpret the high bombardment rate $\sim 3.9$ Gy ago as the tail of a slowly declining, even-more-intense bombardment occurring since the time of formation of the terrestrial planets. However, this seems to be implausible, for several reasons:

i) 600 million years of continual impacts should have left an obvious trace on the Moon. So far, no such trace has been found. The isotopic dating of the samples returned by the various Apollo and Luna missions revealed no impact melt-rock older than 3.92 Gy (Ryder, 1990; Ryder et al. 2002). The lunar meteorites confirm this age limit. The meteorites provide a particularly strong argument because they likely originated from random locations on the Moon (Cohen et al., 2000), unlike the lunar samples collected directly on its surface. A complete resetting of all ages all over the Moon is possible (Hartmann et al., 2000) but highly unlikely, considering the difficulties of completely resetting isotopic ages at the scale of a full planet (Deutsch and Scharer, 1994). The $\mathrm{U}-\mathrm{PB}$ and $\mathrm{Rb}-\mathrm{Sr}$ isochrones of lunar highland samples indicate metamorphic events between 3.85 and 4 Gy ago (Tera et al., 1974). There is no evidence for these isotopic systems being reset by intense collisions between 4.4 and 3.9 Gy.

ii) The old upper crustal lithologies of the Moon do not show the expected enrichment in siderophile elements (in particular the Platinum Group Elements) implied by a period of intense collisions (Ryder et al., 2000) lasting $600 \mathrm{My}$.

iii) If the elevated mass accretion documented in the period around 3.9 Gy is considered to be the tail end of an extended period of even more intense collisions, the Moon should have reached $95 \%$ of its total mass about 4.1 Gy ago instead of 4.5 Gy ago (Ryder, 2002; Koeberl, 2004).

iv) Given the fast dynamical and collisional decay of the population of planetesimals that remain in the vicinity of the Earth's orbit at the end of the accretion process of the terrestrial planets, the formation of two huge impact structures such as the Imbrium and Orientale basins (and probably many more) on the Moon 600 My later implies an implausible initial total mass of solids in the inner solar system (Bottke et al., 2007).

v) The bombardment rate 3.8-3.9 Gy ago (as deduced from the lunar crater record) was probably not intense enough to vaporize the oceans on Earth (Abramov and Mojzsis, 2009). However, if this 
bombardment rate had been the tail of a more intense bombardment, smoothly decaying over time since lunar formation, the ocean evaporation threshold should have been overcome just a few hundreds of millions of years earlier $(\sim 4.2 \mathrm{~Gy}$ ago). This contrasts with the oxygen isotopic signature of the oldest known zircons (age: $4.4 \mathrm{~Gy}$ ), which indicates formation temperatures compatible with the existence of liquid water (Valley et al., 2002).

vi) These same zircons retain secondary over-growths developed after primary core crystallization during their 4.4 Gy long crustal residence times. The rim over-growths can record discrete thermal events subsequent to zircon formation and provide a unique window in crustal processes before the beginning of the terrestrial rock record. In (Trail et al., 2007), all these rim over-growths have been dated to be $\sim 3.9$ Gy old. No (preserved) older rim over-growths, associated to more primordial events, have been found. This suggests that the thermal events were associated to impacts, and that these impacts were concentrated in time about 3.9 Gy ago.

Therefore, it can be concluded that there is strong evidence for a cataclysmic Late Heavy Bombardment event around 3.9 Gy ago. This cataclysm did not just affect the Moon, but has now been clearly established throughout the inner Solar System (Kring and Cohen, 2002). The exact duration of the cataclysm is difficult to estimate, however. Based on the cratering record of the Moon, it lasted between 20 and $200 \mathrm{My}$, depending on the mass flux estimate used in the calculation.

The very existence of an LHB implies that a massive population of planetesimals must have been stored for $\sim 600 \mathrm{My}$ in a stable reservoir, which then was suddenly destabilized. The only intuitive way to do this is that there was a sudden change in the orbital structure of the planets at that time (Levison et al., 2001). In fact, the planets, in particular the giant ones, control the dynamical evolution of the small bodies and determine which reservoir is stable and which unstable. The planetary evolution illustrated in Fig. 8 would do a great job in causing an impact spike, by destabilizing the full outer planetesimal disk when Uranus and Neptune are propelled to their current orbital semi major axes; the asteroid belt would also be partially destabilized when Jupiter and Saturn acquire their current eccentricities and move towards their current orbital separation (Gomes et al., 2005; Minton and Malhotra, 2009). However, in the simulation of Fig. 8 the spike would occur too early to coincide with the LHB spike: we need to find a plausible explanation for which the extraction of the giant planets from their original multiple resonance occurred not after only 2 My (as in Fig. 8), but approximately 600 My later.

The reason for which, in the simulation of Fig. 8 (and in the simulations of Batygin and Brown, 2010), the instability occurs early is that the planets were assumed to be embedded in a planetesimal disk, so that the interaction with said disk was very strong. As pointed out in Gomes et al. (2005), however, this is an unlikely configuration. In fact, the planetesimals that are originally in between the orbits of the giant planets are violently unstable, with a dynamical lifetime well shorter than $1 \mathrm{My}$. Thus, they should have been removed (by colliding with the planets, being ejected onto distant orbits etc.) well before the disappearance of the gas, which typically lasts 


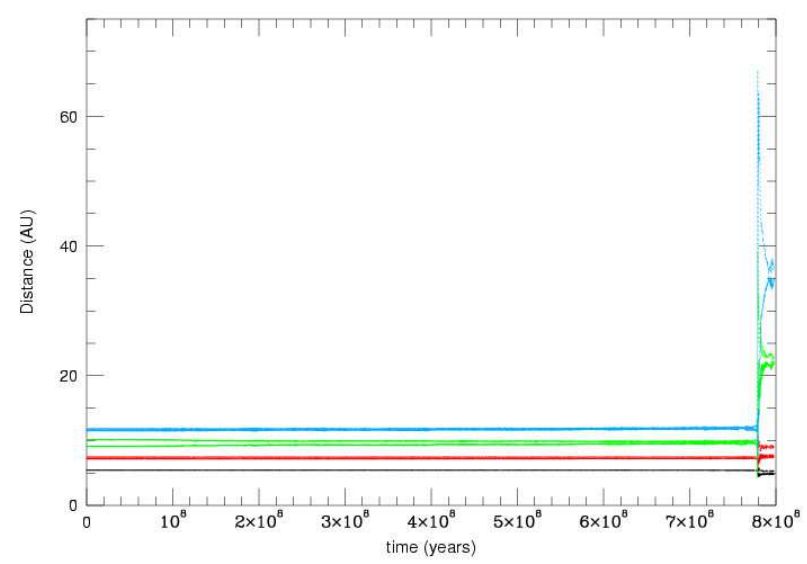

Fig. 9. - The same as Fig. 8 but for a planetesimal disk with an inner edge suitably placed beyond the initial orbit of the outermost planet. Here, the instability is delayed to $\sim 700 \mathrm{My}$, consistent with the timing of the Late Heavy Bombardment of the terrestrial planets.

3-5 My in a protoplanetary disk (Haisch et al., 2001). Then, as said at the beginning of sect. 3, the early removal of these planetesimals should not have changed the orbital configuration of the planets, because the dominant forces exerted by the disk of gas forced the planets to stay in their multiple resonance. Therefore, it is more likely that, at the disappearance of the gas, when $N$ body simulations like that of Fig. 8 become relevant, the planetesimals were only on those orbits whose dynamical lifetime is of the order of the gas-disk lifetime, or longer. This constrains the planetesimals to be in a trans-Neptunian disk, with an inner edge situated at least 1-2 AUs beyond the original semi major axis of Neptune (for simplicity, I call here "Neptune" the planet that is the most distant from the Sun; notice that in some simulations -that of Fig. 8 for instance- the two last planets in order of distance from the Sun switch orbits; in these cases Uranus would have been originally the most distant planet from the Sun).

If the planetesimal disk is beyond the orbit of Neptune, the interactions between the planets and the disk are necessarily much weaker than in the case where the planets are embedded in the disk. In this condition, the instability can occur late, after a time comparable with the LHB chronology, as shown in Fig. 9, I acknowledge, though, that the instability time depends critically on the location of the inner edge of the disk: disks with inner edges slightly closer to Neptune lead to early instabilities, and disks with edges just a bit further give systems that are stable forever 6 . Such extreme sensitivity looks problematic.

All the simulations presented up to this point, however, were simple, because they assumed that

\footnotetext{
${ }^{6}$ The situation was not nearly as sensitive in Gomes et al. (2005), because the planets were not assumed to be in resonance with each other.
} 
the planetesimals do not to interact dynamically with each other. If self-interactions are taken into account, for instance assuming that there are a few 100s Pluto-mass objects in the disk perturbing each other and the other particles, then there is a net exchange of energy between the planets and the disk, even if there are no close encounters between planets and planetesimals. This is because the self-stirring of the disk breaks the reversibility of the eccentricity coupling between planet and planetesimals: the planet eccentricities are damped; however, the evolutions of orbital energy and eccentricity are coupled at second order in the masses (Milani et al., 1987): this produces a drift in the planet's energy. In particular, the planets loose energy, i.e. they try to migrate towards the Sun (Levison et al., 2011). The orbits of the planets tend to approach each other. This is different from the case where planets scatter planetesimals, in which the planetary orbits tend to separate from each other. Remember, though, that the planets are in resonances; so the ratios between their semi major axes cannot change. In response, the planetary eccentricities slowly increase. This eventually drives some planets to pass through secondary or secular resonances, which destabilize the original multi-resonant configuration. The overall evolution is very similar to what is presented in Fig. 9 but now the instability time is late in general: in the simulations of Levison et al. (2011) it ranges from $350 \mathrm{My}$ to over 1 Gy for disks with inner edge ranging from 15.5 to $20 \mathrm{AU}$ (Neptune is at $~ 11.5 \mathrm{AU}$ in these simulations). Unlike the case without self-interactions of disk particles, there is no apparent correlation between instability time and initial location of the inner edge of the disk. This may appear surprising, because the rate of energy exchange between planets and disk decreases with increasing distance of the disk's inner edge. However this dependence is weak, because the planetdisk interaction is a distant interaction (no close encounters are involved). Then, the expected monotonic dependence of the instability time on the disk's distance can be easily erased by the fact that the evolutions of the disk and of the planets are chaotic, which gives a highly sensitive and non-trivial dependence of the results on the initial conditions. The instability time seems to depend weakly also on the number of Pluto-mass scatterers in the disk, provided that this number exceeds a few hundreds.

Together, the papers by Morbidelli et al. (2007) and Levison et al. (2011) build the new version of the "Nice model". This is much superior than its original version (Tsiganis et al., 2005; Gomes et al., 2005) because (i) it removes the arbitrary character of the initial conditions of the planets by adopting as initial configuration one of the end-states of hydro-dynamical simulations and (ii) it removes the sensitive dependence of the instability time on the location of the inner edge of the disk; instead, a late instability seems to be a generic outcome.

In the new Nice model, only $10 \%$ of the simulations which exhibit a global dynamical instability of the planets lead to a stable 4-planets system at the end; in the remaining simulations one or more planets are lost, ejected onto hyperbolic orbits. The fraction of "successful" simulations in the original simulations of Tsiganis et al. (2005) was much higher: $\sim 50 \%$. This is because in the new model the planets are initially in a more compact configuration and therefore their orbital instability is more violent. This low probability of success may suggest that we are still missing something important in our reconstruction of the past history of the solar system, but on the other 
hand it is not so low to reject the model a priori. After all, we have observed only one such system so far! On the positive side, like in Tsiganis et al., when 4 planets survive in the new Nice model, their final orbits are very similar to the real ones: the orbital semi major axes are within 10-15\% of the real values, and the final orbital eccentricities and inclinations are also close (within a factor of 2) to the actual ones. This argues that the model, although certainly not perfect, is probably not too far from reality.

\subsection{The solar system as a debris disk: are LHBs common?}

If our understanding of the evolution of the solar system is (even approximately) correct, there should have been a massive belt of planetesimals outside Neptune's orbit during the first $\sim 600 \mathrm{My}$ of history, i.e. up to the LHB time. This disk, through mutual collisions, should have produced a large amount of dust, generating what it is usually referred to as a "debris disk". It is interesting to investigate how our debris disk would have appeared to an extra-solar observer, and compare the result to the debris disks that we infer around other stars of various ages.

Booth et al. (2009) addressed this question. The intrinsic collision probabilities and mutual velocities among the planetesimals have been computed from the dynamical simulations of the Nice model (in its old version, but this should not make a big difference in this respect). To compute the outcome of the collisional activity, Booth et al. had to assume an initial size distribution. They adopted the size distribution observed in the current Kuiper belt, with the number of objects in each size-bin multiplied by a factor $\sim 1,000$. Thus the initial size distribution contained $\sim 1,000$ Pluto-size bodies, was relatively steep down to $D \sim 100 \mathrm{~km}$, and then turned over to a shallower slope for sizes smaller than this threshold (Bernstein et al., 2004; Fuentes and Holman, 2008). With this assumption, a planetesimal disk with initially $50 M_{\oplus}$ of material looses less than $50 \%$ of its mass in $600 \mathrm{My}$ because of collisional grinding (see also the supplementary material of Levison et al., 2009). This is an important consistency check for the Nice model: in fact, if collisional grinding rapidly removed most of the mass of the disk no matter the initial size distribution, then there would not be enough mass to affect the evolution of the giant planets at the LHB time.

After having computed the dust production rate as a function of time and the orbital and size distributions of the grains, Booth et al. computed the emission of the disk at wavelengths of 24 and 70 microns, relative to the emission of the Sun. The result is illustrated in Fig. 10. It shows that the luminosity of the debris disk should have decayed very slowly, by less than an order of magnitude, during the time preceding the onset of the planetary instability (which occurred at $880 \mathrm{My}$ in the specific simulation of the Nice model used in their calculation). Then, the luminosity should have decayed very rapidly below detectability, as the disk was dynamically dispersed by the planets. The figure also shows, with star symbols, the infrared excess of known stars, as a function of their estimated ages. At 24 microns, only 15\% of the stars younger than 300 My have this kind of excess (Carpenter et al., 2009), and this fraction decreases to a few percent for older stars (Gaspar et al., 2009); at 70 microns, the fraction of stars with detectable infrared excess does not 

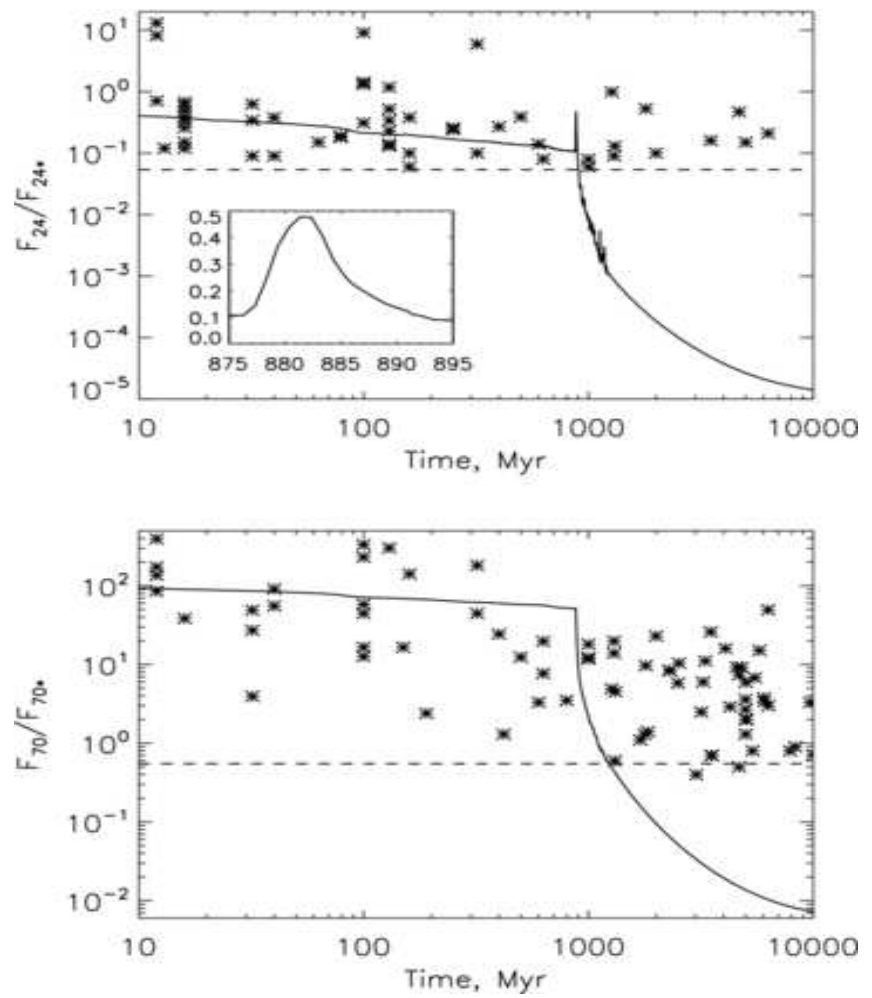

Fig. 10. - The infra-red luminosity of the debris disk of our solar system, according to the Nice model. The solid line shows the luminosity of the disk relative to that of the Sun at 24 microns (top) and 70 microns (bottom). The luminosity decays slowly during the first $880 \mathrm{My}$, when the planets become unstable (in the adopted simulation). Then the luminosity of the disk rapidly decays, as the planetesimals are removed from the solar system. The horizontal dashed line shows the detection limit for an extra-solar observer with our current measurement capabilities. The asterisks represent the observed disks. The window in the lower left corner of the top panel is a magnification of the evolution around the instability time. From Booth et al. (2009).

seem to decay with age (Hillebrand et al., 2008).

A first important conclusion, from the comparison of the estimated brightness of our solar system with the IR-excess of other stars, is that, before the LHB, our debris disk was fairly typical. A second conclusion, from the fact that there is no general tendency for a sudden disappearance of the IR-excess at 70 microns around other stars at about 1 Gy of age, is that a complete dynamical clearing of the planetesimal disk like the one that occurred in our solar system at the LHB time is fairly atypical. From a statistical analysis of the data, Booth et al. estimated that at most $15 \%$ of the extra-solar systems undergo such a late dynamical clearing.

The fact that a late complete dynamical clearing of the planetesimal disk is a rare event should not be a big surprise. In fact, it is clear from what has been said above that the events described in the Nice model depend on two specific properties that not many planetary systems might have in common with our own. First, the planets in our system did not migrate permanently into 
the inner solar system; instead, they remained or returned near their birth places, i.e. adjacent to the planetesimal disk that generated them. Thus, when their orbits changed at the time of the instability, they strongly affected the disk. If the planets had migrated close to the Sun and had remained there, they would have lost contact with the distant planetesimal disk. Even if the planets had become unstable in the inner solar system, probably the distant disk would not have been dynamically cleared. Second, the planetesimal disk of the solar system was small, presumably truncated at $\sim 30 \mathrm{AU}$ (Gomes et al., 2004). If the planetesimal disk had been extended to much larger distances, the dynamical instability and the migration of Neptune would have probably cleared the disk up to 50-100 AU; beyond this threshold the disk would have remained massive and would have continued to produce dust.

Coming back to Fig. 10, the little spike in the disk's brightness visible at $880 \mathrm{My}$ in the upper plot (magnified in the box in the bottom left corner) is the signature of the LHB event, due to a burst in collisional activity that occurs as the disk starts to be dispersed and its orbital excitation suddenly increases. As one can see, the spike is not prominent enough to make the disk stand out of the natural distribution of brightness of disks of different masses (suggested by the dispersion of the observations reported on the top panel of Fig. 10). The Booth et al. calculation, however, does not account for the huge flux of comets into the inner solar system that should have occurred during the disk dispersal: these comets should have liberated a great amount of dust once inside a few AUs from the Sun. Nesvorny et al. (2010) accounted for this effect: they estimated that the inner zodiacal cloud should have been more than $10^{4}$ times brighter during the LHB epoch. As the current infrared excess at 24 microns of the zodiacal cloud is $2 \times 10^{-4}$ (Kelsall et al., 1998), the excess at the LHB time at this wavelength was probably of order $2-10$. This kind of excess is comparable to the upper envelope of the observations. Thus, the conclusion is that luminosity bursts associated to LHB events, totally invisible at 70 microns (cold dust), start to be detectable at 24 microns (hot dust), although they can still be confused with the luminosity of massive disks undergoing gradual collisional grinding. Therefore, the identification of systems that might be undergoing an LHB event at the current time requires a case by case analysis at multiple wavelengths, as done for instance in Wyatt et al. (2007).

\section{Terrestrial planets}

Up to this point, this chapter was focussed on the formation and evolution of giant planets. I now extend briefly the discussion to the case of the terrestrial planets.

Terrestrial planets are expected to accrete in the inner part of a proto-planetary disk, closer to the star than the snowline position. Because of the absence of ices and of the relatively small mass contained in the inner portion of the disk, the largest objects formed by the processes of runaway and oligarchic growth (see Sect. 2.1) are expected to have masses of about a Lunar to a Martian mass (Weidenshilling et al., 1997). These bodies are called planetary embryos to distinguish them from the much more massive planetary cores that are the precursors of the giant planets. They 

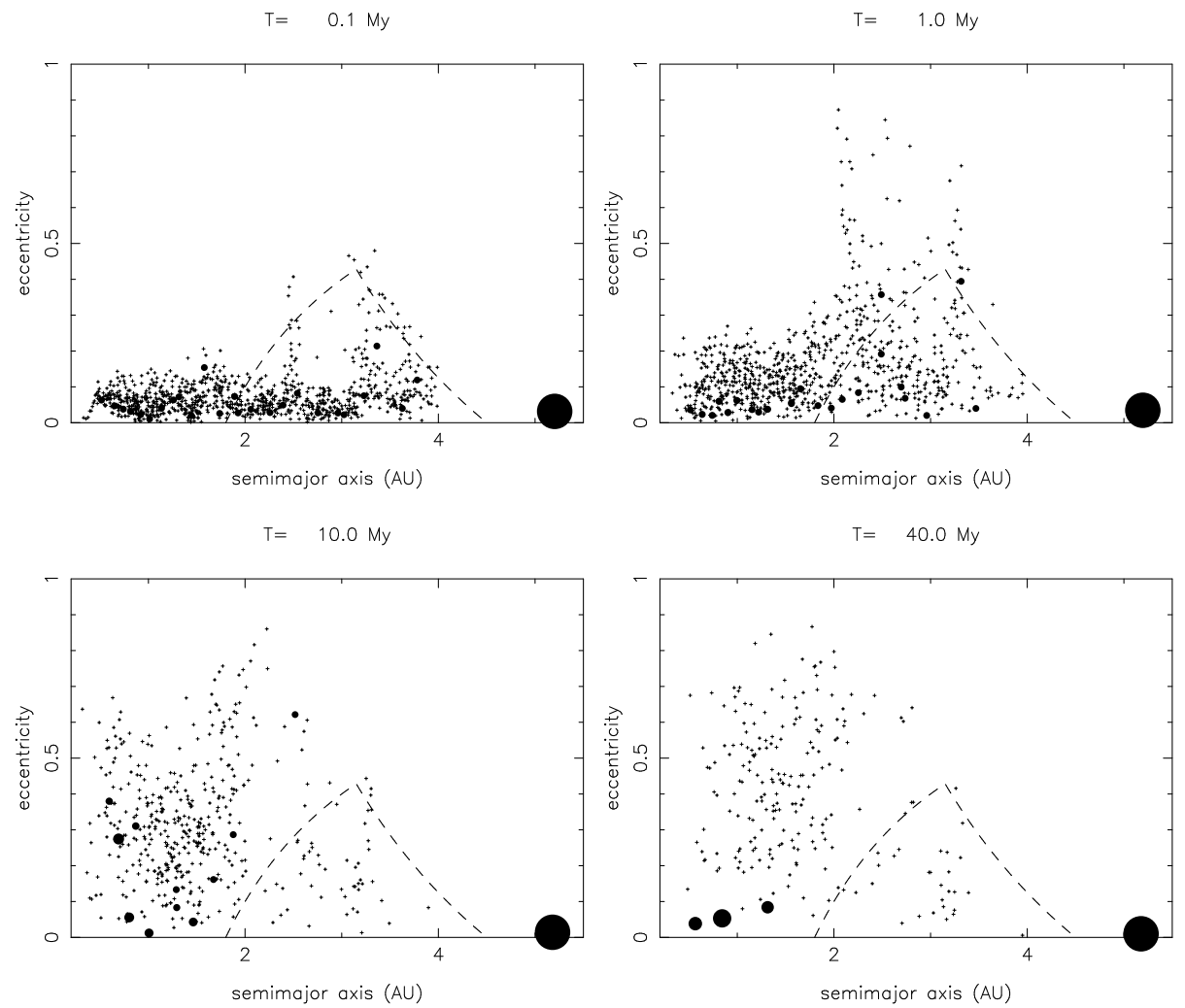

Fig. 11. - Snapshots of the terrestrial planets formation process, from O'Brien et al., (2006). Each panel shows the eccentricity vs. semi major axis distribution of planetary embryos (filled circles) and planetesimals (crosses) at the reported time. The size of each filled circle is proportional to the cubic root of the mass of the corresponding embryo. The big filled ball at $\sim 5.2 \mathrm{AU}$ represents Jupiter. The dashed curves show the current boundaries of the asteroid belt. Notice the formation of three terrestrial planets in this simulation, the biggest of which is approximately one Earth mass. In the asteroid belt no terrestrial planet is formed. All embryos have left the asteroid belt region and only a small fraction of the initial planetesimals reside there on excited orbits. In these simulations all collisions are supposed to be accretional. This approximation has been recently removed by Kokubo and Genda (2010), who considered a database of collisions simulated by the SPH method (like in Agnor and Asphaug, 2004; Asphaug et al., 2006) to determine how much mass is accreted or ejected in each collsional event. The final results, though, show very little differences with respect to the simulations that treat all collisions as $100 \%$ accretional.

incorporate about $50 \%$ of the mass of solids in the inner disk, the rest remaining in asteroid-sized planetesimals.

The evolution of the system of embryos and planetesimals has been the object of many simulations using $N$-body integrations (Chambers and Wetherill, 1998; Agnor et al., 1999; Chambers, 2001; Raymond et al., 2004, 2005, 2006, 2007, 2009b; O'Brien et al., 2006; Kenyon and Bromley, 2006; Kokubo et al., 2006; Thommes et al., 2008; Morishima et al., 2010; Kokubo and Genda, 2010). All studies agree on the basic aspects of the terrestrial planet accretion process, although they may differ in the details. At the disappearance of the gas, the system of embryos becomes 
violently unstable, due to the mutual interactions among the embryos themselves and to the "external" perturbations from the giant planets. The orbits of the embryos begin to intersect and accretional collisions between embryos start to occur. This produces a smaller number of more massive objects (see Fig. 11), which are eventually identified with the terrestrial planets.

Concerning the planetesimals, a fraction of them contributes to the growth of the planets by colliding with the embryos. The majority of the planetesimals, however, are dispersed onto orbits with large eccentricities and inclinations. In this process (the "dynamical friction" mentioned in Sect. 3.1), they damp the eccentricities and inclinations of the growing planets, which eventually sets the system into a stable configuration, with the most massive planets on the least eccentric orbits.

This scenario for the formation of the terrestrial planets of our solar system has several strong points:

- A system of 2-5 planets is typically formed. The efficiency of the accretion process is about $50 \%$. Thus, starting with $\sim 5 M_{\oplus}$ in embryos and planetesimals typically produces a couple of planets of about an Earth mass each (Chambers, 2001). The final orbits of the terrestrial planets produced in the simulations are comparable to those of the real terrestrial planets of our solar system, if the dynamical friction process is properly taken into account (O'Brien et al., 2006).

- Quasi-tangent collisions of Mars-mass embryos onto the proto-planets are quite frequent (Agnor et al., 1999). These collisions are expected to generate a disk of ejecta around the proto-planets (Canup and Asphaug, 2001), from which a satellite is likely to accrete (Canup and Esposito, 1996). This is the standard, generally accepted scenario for the formation of the Moon.

- The accretion timescale of the Earth analog in the simulations is $30-100 \mathrm{My}$. This is in the good ballpark with the chronology of Earth accretion as indicated by radioactive chronometers, which still has a comparable uncertainty (Kleine et al., 2009).

- In many/most simulations, terrestrial planets do not form in the asteroid belt. Instead, all the embryos are removed by mutual interactions and perturbations from Jupiter. A small fraction (a few percent) of the planetesimal population is left behind on stable asteroid-belt orbits, with eccentricities and inclinations comparable to those of the real asteroids (Petit et al., 2001; O’Brien et al., 2007).

- A significant fraction $(\sim 10-20 \%)$ of the mass of the terrestrial planets is accreted from the outer part of the asteroid belt, which provides a formidable mechanism to explain the delivery of water to the Earth (Morbidelli et al., 2000; Raymond et al., 2004, 2007).

On the other hand, this scenario has a major problem: the planet formed in the simulations at the location of Mars is typically too massive (Chambers, 2001; Raymond et al., 2009; Hansen, 
2009; Morishima et al., 2010). Mars is an oddity not only for what it concerns its mass, but also its accretion timescale: in fact, it formed in a few millions of years only, like asteroids, i.e. much faster than the Earth (Dauphas and Pourmand, 2011).

There does not seem to be a simple solution to the Mars problem (Raymond et al., 2009). Hansen (2009) argued convincingly that a correct mass distribution of the terrestrial planets, with an Earth/Mars mass ratio of $\sim 10$, can be achieved only if the initial disk of embryos and planetesimals is assumed to have an outer edge at about $1 \mathrm{AU}$. The problem is how to justify such an edge and how to reconcile this with the evidence that asteroids exist in the 2-4 AU range. The simulations that assume Jupiter and Saturn initially on orbits with their current separation in semi major axis but eccentricities $2-3$ times larger, do produce very rapidly an effective edge at $\sim 1.5 \mathrm{AU}$ in the distribution of embryos and planetesimals (Raymond et al., 2009; Morishima et al., 2010) and result in a somewhat small "Mars". However, this initial configuration of the giant planets is inconsistent with our understanding of their orbital evolution through the history of the solar system, described above (see Sects. 2.4, 3.2 and 3.3). Moreover, none of these simulations are without problems: Mars is often not small enough, the final distribution of bodies in the asteroid belt is not good, water is not delivered to the terrestrial planets etc..

\subsection{Linking giant planet migration to terrestrial planet accretion: the Grand Tack scenario}

The result by Hansen motivated Walsh et al. (2011) to look in more details at the possible orbital history of the giant planets and their ability to sculpt the disk in the inner solar system. For the first time, the giant planets were not assumed to be on static orbits (even if different from the current ones); instead Walsh et al. studied the co-evolution of the orbits of the giant planets and of the precursors of the terrestrial planets, during the era of the disk of gas.

Walsh et al. envisioned the following scenario, based on the considerations reported in Sect. 2.2. first, Jupiter migrated inwards while Saturn was still growing; then, when Saturn reached a mass close to its current one, it started to migrate inwards more rapidly than Jupiter, until it captured the letter in the $2 / 3$ resonance; finally the two planets migrated outwards until the complete disappearance of the disk of gas. The extent of the inward and outward migrations cannot be estimated a priori, because they depend on properties of the disk and of giant planet accretion that are unknown, such as: the time-lag between Jupiter and Saturn formation, the speed of inward migration (depending on disk's viscosity), the speed of outward migration (depending on disk's scale height), the time-lag between the capture in resonance of Jupiter and Saturn and the photoevaporation of the gas. However, the extent of the inward and outward migrations of Jupiter can be deduced by looking at the resulting structure of the inner solar system. In particular, Walsh et al. remarked that a reversal of Jupiter's migration at 1.5 AU would provide a natural explanation for the existence of the outer edge at $1 \mathrm{AU}$ of the inner disk of embryos and planetesimals, required to produce a small Mars (see Fig. 12). Because of the prominent inward-then-outward migration 


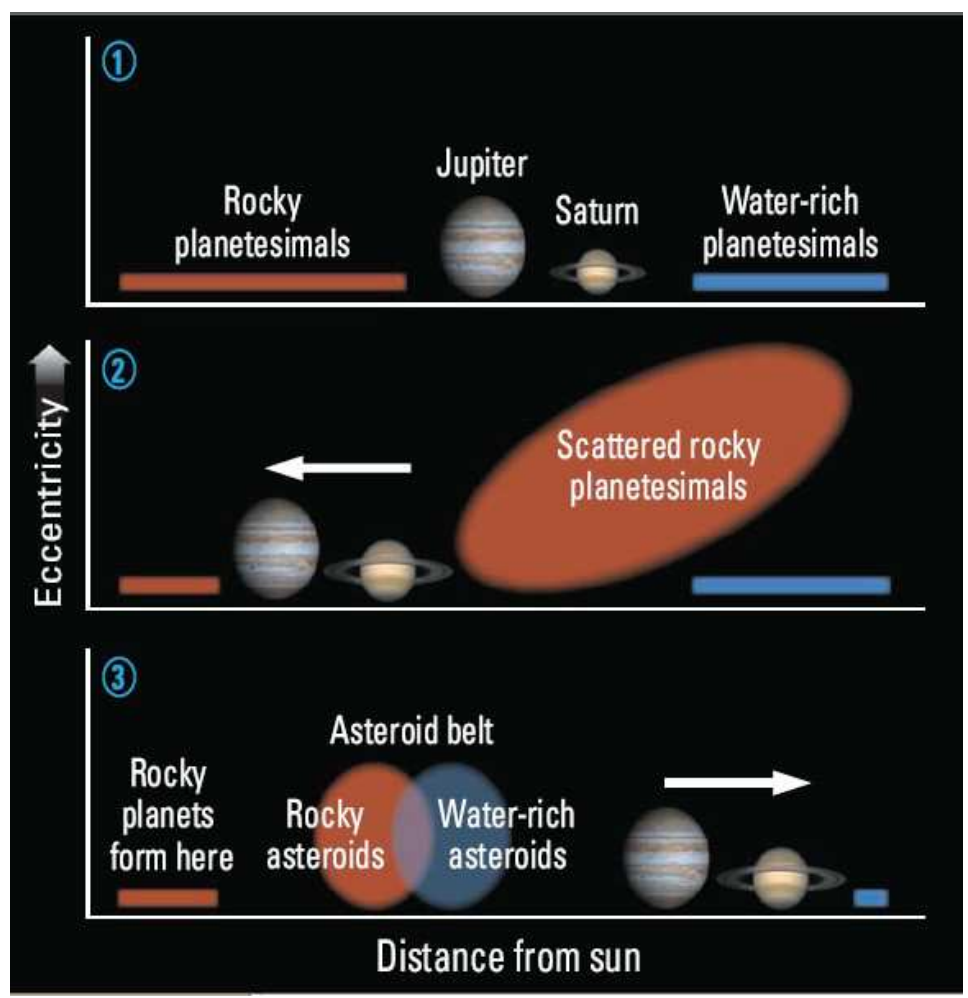

Fig. 12. - Sketch of the "Grand Tack scenario". The three panels depict subsequent steps in the evolution of the system. Form "NEWS\&ANALYSIS": Science, 332, 1255 (2011).

of Jupiter that it assumes, Walsh et al. scenario is nicknamed "Grand Tack".

Several giant extra-solar planets have been discovered orbiting their star at a distance of 1-2 $\mathrm{AU}$, so the idea that Jupiter was sometime in the past at 1.5 AU from the Sun is not shocking by itself. A crucial diagnostic of this scenario, though, is the survival of the asteroid belt. Given that Jupiter should have migrated through the asteroid belt region twice, first inwards, then outwards, one could expect that the asteroid belt should now be totally empty. However, the numerical simulations by Walsh et al. show that the asteroid belt is first fully depleted by the passage of the giant planets, but then, while Jupiter leaves the region for the last time, it is re-populated by a small fraction of the planetesimals scattered by the giant planets during their migration. In particular, the inner asteroid belt is dominantly re-populated by planetesimals that were originally inside the orbit on which Jupiter formed, while the outer part of the asteroid belt is dominantly re-populated by planetesimals originally in between and beyond the orbits of the giant planets (see Fig. 12).

Assuming that Jupiter accreted at the location of the snow line, it is then tempting to identify the planetesimals originally closer to the Sun with the un-hydrous asteroids of S-type and those originally in between and beyond the orbits of the giant planets with the "primitive" C-type asteroids. With this assumption, the Grand Tack scenario explains the physical structure of the 
asteroid belt probably better than any other previous model. In fact, the asteroid belt is characterized by a radial gradient in asteroid spectroscopic types (Gradie and Tedesco, 1982): the inner belt is dominated S-type (usually considered to be the parent bodies of ordinary chondrites; Binzel et al., 1996), the outer belt by C-type asteroids (usually considered to be the parent bodies of carbonaceous chondrites; Burbine, 2000), although there is a significant overlapping between the distributions of these different types of asteroids. It is difficult to explain the differences between ordinary chondrite and carbonaceous chondrite parent bodies if they had both formed in the asteroid belt region, given that they are coeval (Villeneuve et al., 2009) and that the radial extent of the asteroid belt is small ( 1 AU only). Instead, if ordinary and carbonaceous chondrite parent bodies have been implanted into the asteroid belt from originally well separated reservoirs, as in the Grand Tack scenario, the differences in physical properties are easier to understand in the framework of the classical condensation sequence. The origin of C-type asteroids from the giant planet region would also explain, in a natural way, the similarities with comets that are emerging from recent observational results and sample analysis (see sect 7 of the supplementary material of Walsh et al., 2011, for a in-depth discussion). The small mass of the asteroid belt and its eccentricity and inclination distribution are also well reproduced by the Grand Tack scenario.

This scenario also explains why the accretion timescales of Mars and the asteroids are comparable (Dauphas and Pourmand, 2011). In fact, the asteroids stopped accreting when they got dispersed and injected onto excited orbits of the main belt; Mars stopped accreting when the inner disk was truncated at $1 \mathrm{AU}$ and the planet was pushed beyond this edge by an encounter with the proto-Earth (Hansen, 2009). In the Grand Tack scenario these two events coincide, and mark the time of the passage of Jupiter through the inner solar system.

All these results make the Grand Tack scenario an appealing comprehensive model of terrestrial planet formation and argue strongly in favor of an evolution of the giant planets of our solar system like that sketched in the right panel of Fig. 6 .

\subsection{Terrestrial planets in extra-solar systems}

Given that the architecture of the giant planets of our solar system is far from being typical around other stars, it is interesting to investigate the dependence of the terrestrial planet accretion process on the properties of giant planets, across a wide range of parameters.

From various simulations (Levison and Agnor, 2003; Raymond et al., 2004; 2006), it turns out that the outcome of the terrestrial planet formation process has a weak dependence on the mass of the giant planets. Obviously, the terrestrial planets cannot form in the vicinity of giants. So, if the giant planets are closer to the star than Jupiter, they leave to the terrestrial planets a narrower niche to form inside. Instead, the process of terrestrial planets accretion is very sensitive on the eccentricities of the giant planets. Large eccentricities of the giant planets force large eccentricities on embryos and planetesimals. As a result, the final terrestrial planets will be more eccentric; 
consequently they will have a larger separation in semi major axis, will be less numerous and more massive compared to a simulation where the same giant planets are on circular orbits. Moreover, the planetesimals originally in the vicinity of the giant planets (presumably rich in water and other volatiles) are more likely to be ejected from the system than to collide with the terrestrial planets, if the giant planets are eccentric (Chambers and Cassen, 2002; Raymond et al., 2004). So, the resulting terrestrial planets are expected to be water-poor.

The works quoted above assumed giant planets on "fixed" orbits. We know now that the giant planets can have evolutions that lead them to change their orbits, through migration and/or dynamical instabilities. It is interesting to explore how the terrestrial planets, during and after their formation, respond to these changes.

The effect of a Jupiter-mass planet migrating through the disk towards a "hot-Jupiter" orbit has been investigated in Fogg and Nelson $(2005,2007)$ and Raymond et al. (2006b). These studies showed that a large fraction of the disk's solid mass survives the inward migration of the giant planet in two ways: (i) planetesimals are captured into mean motion resonances interior to the orbit of the giant planet and, by mutual collisions, give origin to massive terrestrial planets; (ii) planetesimals are scattered into external orbits, where gas drag re-circularizes their orbits; the standard terrestrial planet formation process then resumes. Thus, the wide-spread expectation that terrestrial planets could not exist in systems with a hot Jupiter is not correct and future searches for extra-solar terrestrial planets should not disregard these systems a priori.

\subsection{Terrestrial planets evolution during giant planets instabilities}

The issue of terrestrial planet evolution during giant planets instabilities deserves a whole section by itself. As usual, I start with a description of our understanding of what happened in the solar system.

As we have seen in Sect. 3.3, we believe that the giant planets of our solar system passed through a phase of orbital instability $\sim 3.9$ Gy ago, i.e. well after the formation of the terrestrial planets (which ended $\sim 4.5$ Gy ago; Kleine et al., 2009). During this instability phase, close encounters between the giant planets occurred; the orbits of the giant planets became eccentric and their separation in semi major axis increased towards the current values. During this period of chaotic evolution, a wide variety of orbital histories are possible. We may, however, classify the orbital histories in two classes: those in which Jupiter is not involved in close encounters with another planet (only Saturn, Uranus and Neptune have encounters with each other) and those in which Jupiter has encounters with Uranus and/or Neptune (nicknamed below the jumping-Jupiter class). The two classes give a very different evolution of the orbital separation of Jupiter and Saturn.

In the first class of evolutions, the increase in orbital separation between Jupiter and Saturn is due to planetesimal-driven migration. In fact, if Saturn scatters an ice giant (Uranus or Neptune) 


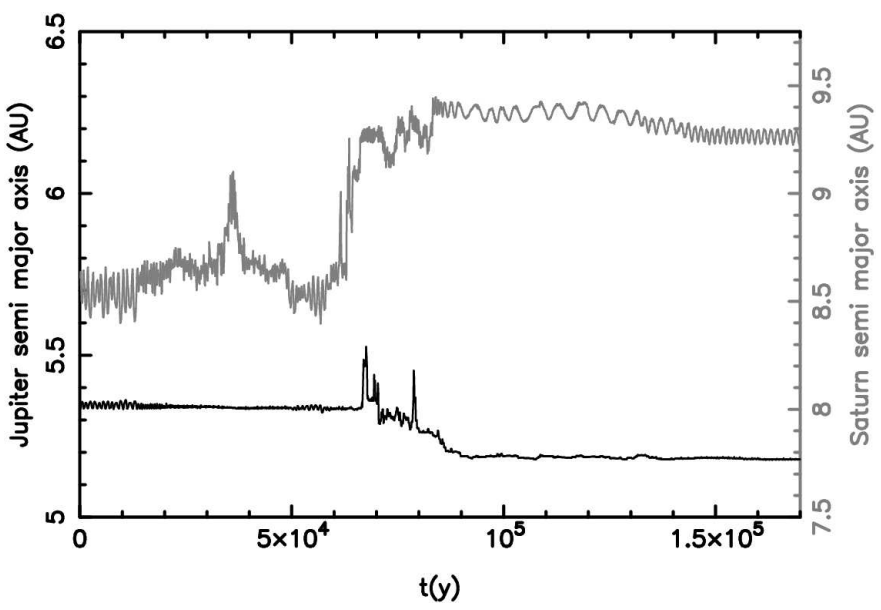

Fig. 13. - An example of "jumping-Jupiter evolution". The black and gray curves show the evolution of the semi major axes of Jupiter and Saturn, reported in the left-side and right-side vertical scales, respectively. The stochastic behavior is caused by encounters with a Uranus/Neptune-mass planet, originally placed the third in order of increasing distance from the Sun (not shown here). Time $t=0$ here is arbitrary and corresponds to the onset of the phase of planetary instability. The full evolution of the planets, which lasts 4.6 My, is illustrated in Fig. 4 of Brasser et al. (2009). All giant planets survived the full 4.6 My simulation on stable orbits, quite similar to those of the real planets of the Solar System.

while Jupiter does not, necessarily Saturn has to scatter the ice giant outwards and recoil towards the Sun. So, planetary encounters in this class of evolutions lead to a reduction of the orbital separation of Jupiter and Saturn and planetesimal-driven migration is the only mechanism that can increase it. In the jumping-Jupiter class of evolutions, most of the increase in orbital separation between the two gas giants is instead due to planetary encounters. In fact, if Jupiter has an encounter with an ice giant, said ice giant, given that it is beyond the orbit of Saturn both at the beginning and at the end of the evolution, must be first scattered inwards by Saturn and then be scattered outwards by Jupiter. Thus, Saturn recoils outwards and Jupiter inwards, which increases the orbital separation between Jupiter and Saturn.

In summary, both classes of evolutions lead to an increase in the orbital separation of Jupiter and Saturn, but the big difference is in the timescale on which this separation occurs. Planetesimaldriven migration is relatively slow: it forces the orbital separation to evolve exponentially as $\Delta a(t)=$ $\Delta a_{\text {current }}-\Delta_{0} \exp (-t / \tau)$, with $\tau \sim 5-10$ My (the characteristic lifetime of planetesimals crossing the orbits of the giant planets, such as the Centaur objects; Tiscareno and Malhotra, 2003; DiSisto and Brunini, 2007; Bailey and Malhotra, 2009). Conversely, the phase of planetary encounters is short, so that in the jumping-Jupiter class the orbital separation of Jupiter and Saturn increases in less than $10^{5} \mathrm{y}$ (see Fig. 13).

The increase in orbital separation between Jupiter and Saturn changes the secular frequencies of the orbits of these planets. If the divergent migration of Jupiter and Saturn occurs on a timescale of a few millions of years, as in the case of planetesimal-driven migration, the orbits of the terrestrial 


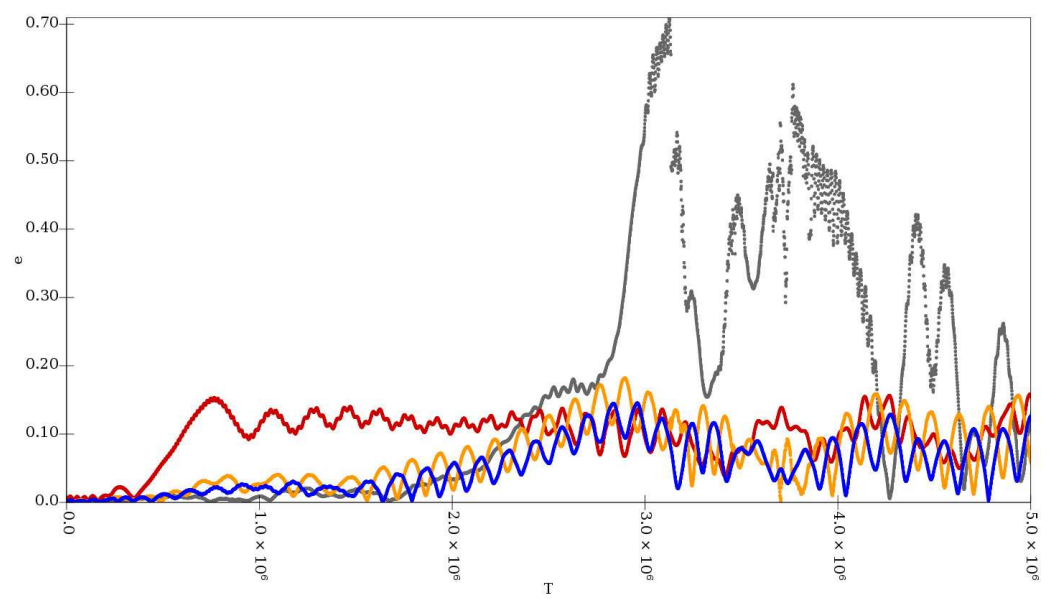

Fig. 14.- The evolutions of the eccentricities of Mars (red), Earth (blue), Venus (orange) and Mercury (grey), during planetesimal-drive migration of Jupiter and Saturn. Here the orbital separation between the two gas giants increases as $\Delta a_{\text {current }}-\Delta_{0} \exp (-t / \tau)$, with $\Delta_{0}=1.1 \mathrm{AU}$ and $\tau=5 \mathrm{My}$.

planets are quite strongly excited in eccentricity (Brasser et al., 2009). Even if starting from circular orbits, the Earth and Venus acquire orbits whose eccentricity oscillations exceed 0.15, i.e. twice as much as in the real solar system; Mercury is destabilized (Fig. 14). This happens because the frequency of precession of the perihelion of Jupiter's orbit, denoted by $g_{5}$, decreases and, in sequence, it becomes equal to those of Mars, Earth, Venus and Mercury $\left(g_{4}, \ldots, g_{1}\right)$ respectively; every time that a frequency of a terrestrial planet $g_{k}$ is equal to $g_{5}$ a secular resonance occurs and the eccentricity of the corresponding planet is strongly affected. Similarly, most of the asteroids in the inner part of the asteroid belt are destabilized while the precession frequency of the perihelion of the orbit of Saturn $\left(g_{6}\right)$ decreases; consequently, the final orbital distribution of the asteroids is incompatible with the one currently observed (Morbidelli et al., 2010). In the jumping-Jupiter class of evolutions, conversely, these problems do not exist because the divergent migration of Jupiter and Saturn -and the consequent decrease of $g_{5}$ and $g_{6}-$ are so fast that the eccentricities of asteroids and terrestrial planets have no time to be seriously affected. Thus, the terrestrial planets can have at the end orbital eccentricities as small (or even smaller) than the current ones, depending on their initial conditions (Brasser et al., 2009) and the asteroid belt preserves roughly the orbital structure acquired during terrestrial planets formation (Morbidelli et al., 2010).

The conclusion is that the real evolution of the giant planets of our solar system had to be of the jumping-Jupiter class; otherwise the orbit of the Earth, and the structure of the inner solar system in general, would be substantially different from what they are now.

There is no doubt that many evolutions of the giant planets can be fatal for the formation or the evolution of habitable terrestrial planets. Take the giant extra-solar planets discovered so far: most of them have very eccentric orbits, which are thought to be the product of a violent instability occurred in the original planetary system, which led to close encounters between giant planets (see Sect. 2.3). Raymond and Armitage (in preparation) show that when giant planets 
acquire similarly large eccentricities, the terrestrial planets in the system are forced to evolve onto orbits with extreme eccentricities: many of them collide with the central star, or start to intersect the orbits of the giant planets and are then rapidly ejected onto hyperbolic orbits. Those terrestrial objects which manage to survive, if any, do so on orbits with eccentricities that can be hardly compatible with habitable worlds. Thus, the existence of an habitable Earth in our system is possible only because our giant planets remained on orbits with exceptionally small eccentricities compared to the orbits of extra-solar planets.

\section{Conclusions}

This chapter discussed the evolution of planetary systems. The emphasis has been put on the evolution of our solar system. Our team effort, over the last 10 years, has been to reconstruct the history of our system using computer simulations and taking advantage of all possible detailed constraints (the orbits of the planets, the architecture of the populations of small bodies, radioactive chronologies for terrestrial planet formation, crater records etc.). Although imperfect, I think that our view of the evolution of the solar system, since the completion of giant planets formation, has reached a quite satisfactory level of coherence. Conversely, the phase of accretion of the giant planets remains poorly modeled.

According to our understanding, the evolution of the solar system was characterized by three main "eras". In the gas-disk era, Jupiter had a wide-range radial migration. It first migrated inwards; then, when it was at about 1.5 AU from the Sun, it got caught in resonance with Saturn and, given the Jupiter/Saturn mass-ratio, it started to migrate outwards (Walsh et al., 2011). This inward-then-outward migration explains why we do not have a "hot (or warm) Jupiter" in our solar system. It also left indelible traces in the inner solar system, particularly in the physical structure of the asteroid belt and the small mass of Mars. As a result of the outward migration of Jupiter, the four giant planets acquired a multi-resonant configuration, in which each planet was in a mean-motion resonance with its neighbor. The orbits of the giant planets were at the time much closer to each other than they are now, and had significantly smaller eccentricities and inclinations.

At the disappearance of the gas, the system entered in the planetesimal-disk era. The Earth and Venus completed their accretion from a disk of planetary embryos and planetesimals that inherited an outer edge at $1 \mathrm{AU}$ from the earlier incursion of Jupiter into the inner solar system. The accretion of Mars and of the asteroids was frozen. Instead, a massive disk of planetesimals persisted outside the orbit of the outermost giant planet. Its internal collisional activity produced a debris disk comparable to those observed around $\sim 15 \%$ of main sequence stars. Meanwhile, the gravitational interactions between the giant planets and this disk, slowly modified the resonant orbit of the former. Eventually, $\sim 600 \mathrm{My}$ later, the giant planets became unstable, as a result of these slow orbital modifications. The chaotic phase that followed reshuffled the structure of the outer solar system: the giant planets acquired their current orbits; most of the distant planetesimal disk was dispersed and the Kuiper belt is what remains today of that disk (Levison et al., 2008; 
Batygin et al., 2011). Many asteroids also got released from the asteroid belt. All these destabilized small bodies caused the Late Heavy Bombardment of the terrestrial planets (Bottke et al., 2011).

With this profound re-organization, the solar system entered into the current era, lasting since $\sim 3.8$ Gy ago, in which it did not suffer any further significant change.

If this story is true, then the evolution of our solar system was defined by a sequence of specific features. For instance, the mass ratio between Jupiter and Saturn prevented migration towards the Sun; the late formation of the giant planets relative to the gas disk lifetime prevented Saturn to grow more massive than Jupiter; the instability phase that characterized the giant planets resulted in a jumping-Jupiter evolution, which prevented secular resonances to interfere with the orbits of the terrestrial planets, etcetera. It was then natural in this chapter to discuss what would have happened if these events had not occurred, or if they had occurred differently. This led me to address the origin of the diversity of planetary systems observed around other stars. The possible lines of evolutions that I have described certainly do not exhaust all possibilities: I'm sure that Nature has much more fantasy than we have. However, they show that the evolution of a planetary system, like the weather on Earth, is so sensitive on initial and environmental conditions that a huge variety of outcomes is possible, even starting from similar situations.

A frequently asked question, at this point, is whether one can predict the probability that a system evolves towards one state or another. The answer at this time, unfortunately, is no. Our understanding of the first stages of planet formation is too limited; moreover we don't know well enough the initial conditions, i.e. the properties of proto-planetary disks. So, we cannot say which kinds of planetary systems could be formed and with which probabilities; without this information, we cannot estimate the probabilities of the subsequent possible evolutions. For instance, we don't know why Jupiter and Saturn formed like they are, instead of having different masses or having additional gas giant companions. In other words, we can try to reconstruct the evolution of the solar system using all available clues and constraints, but we are not able to "predict" our solar system, a-priori. I'm not sure that we will ever be able to do so. I think this field has a parallel with geology. Geologists are able to reconstruct the complicated history of our continents with an amazing precision, but they are not able to say what is the probability that a terrestrial planet develops continents with the properties of our own ones. We have to acknowledge that, at this time, field of "origins" in planetary science is essentially a descriptive discipline. As such, it is led by observations and interpreted by theoretical models, not the other way around.

I am grateful to the Helmholtz Alliance's 'Planetary evolution and life' and the French National Programme for Planetary Science, for substantial financial support. I wish to thank all the people with whom I worked over these fabulous years in the quest for a better understanding of the evolution of planetary systems and of our solar system in particular. I'm grateful to P. Michel, A. Crida, K. Walsh and an anonymous reviewer, for carefully reading a first draft of this chapter and for providing useful comments and suggestions. 


\section{References}

- Abramov, O., Mojzsis, S. J. 2009. Microbial habitability of the Hadean Earth during the late heavy bombardment. Nature 459, 419-422.

- Adams, F. C., Laughlin, G. 2003. Migration and dynamical relaxation in crowded systems of giant planets. Icarus 163, 290-306.

- Agnor, C. B., Canup, R. M., Levison, H. F. 1999. On the Character and Consequences of Large Impacts in the Late Stage of Terrestrial Planet Formation. Icarus 142, 219-237.

- Agnor, C., Asphaug, E. 2004. Accretion Efficiency during Planetary Collisions. The Astrophysical Journal 613, L157-L160.

- Alibert, Y., Mordasini, C., Benz, W. 2004. Migration and giant planet formation. Astronomy and Astrophysics 417, L25-L28.

- Asphaug, E., Agnor, C. B., Williams, Q. 2006. Hit-and-run planetary collisions. Nature 439, 155-160.

- Bailey, B. L., Malhotra, R. 2009. Two dynamical classes of Centaurs. Icarus 203, 155-163.

- Baldwin, R. B. 2006. Was there ever a Terminal Lunar Cataclysm?. With lunar viscosity arguments. Icarus 184, 308-318.

- Barge, P., Sommeria, J. 1995. Did planet formation begin inside persistent gaseous vortices?. Astronomy and Astrophysics 295, L1-L4.

- Barnes, R., Greenberg, R. 2006. Stability Limits in Extrasolar Planetary Systems. The Astrophysical Journal 647, L163-L166.

- Baruteau, C., Masset, F. 2008. On the Corotation Torque in a Radiatively Inefficient Disk. The Astrophysical Journal 672, 1054-1067.

- Batygin, K., Brown, M. E. 2010. Early Dynamical Evolution of the Solar System: Pinning Down the Initial Condition of the Nice Model. ArXiv e-prints arXiv:1004.5414.

- Batygin, K., Brown, M. E. and Fraser, W.C. 2011. In-situ Formation of the Cold Classical Kuiper Belt. ApJ, sumbitted.

- Bernstein, G. M., Trilling, D. E., Allen, R. L., Brown, M. E., Holman, M., Malhotra, R. 2004. The Size Distribution of Trans-Neptunian Bodies. The Astronomical Journal 128, 1364-1390.

- Binzel, R.P., Bus, S.J., Burbine, T.H., Sunshine, J.M. 1996. Spectral Properties of Near-Earth Asteroids: Evidence for Sources of Ordinary Chondrite Meteorites. Science 273, 946-948. 
- Bitsch, B., Kley, W. 2010. Orbital evolution of eccentric planets in radiative discs. Astronomy and Astrophysics 523, A30.

- Bodenheimer, P., Hubickyj, O., Lissauer, J. J. 2000. Models of the in Situ Formation of Detected Extrasolar Giant Planets. Icarus 143, 2-14.

- Boley, A. C. 2009. The Two Modes of Gas Giant Planet Formation. The Astrophysical Journal 695, L53-L57.

- Booth, M., Wyatt, M. C., Morbidelli, A., Moro-Martín, A., Levison, H. F. 2009. The history of the Solar system's debris disc: observable properties of the Kuiper belt. Monthly Notices of the Royal Astronomical Society 399, 385-398.

- Boss, A. P. 2000. Possible Rapid Gas Giant Planet Formation in the Solar Nebula and Other Protoplanetary Disks. The Astrophysical Journal 536, L101-L104.

- Boss, A. P. 2001. Formation of Planetary-Mass Objects by Protostellar Collapse and Fragmentation. The Astrophysical Journal 551, L167-L170.

- Boss, A. P. 2002. Stellar Metallicity and the Formation of Extrasolar Gas Giant Planets. The Astrophysical Journal 567, L149-L153.

- Bottke, W. F., Levison, H. F., Nesvorný, D., Dones, L. 2007. Can planetesimals left over from terrestrial planet formation produce the lunar Late Heavy Bombardment?. Icarus 190, 203-223.

- Bottke, W. F., Vokrouhlicky, D., Minton, D., Nesvorny, D., Brasser, R., Simonson, B. 2011. The Great Archean Bombardment, or the Late Late Heavy Bombardment. Lunar and Planetary Institute Science Conference Abstracts 42, 2591.

- Brasser, R., Morbidelli, A., Gomes, R., Tsiganis, K., Levison, H. F. 2009. Constructing the secular architecture of the solar system II: the terrestrial planets. Astronomy and Astrophysics 507, 1053-1065.

- Burbine, T.H., Binzel, R.P., Bus, S.J., Buchanan, P.C., Hinrichs, J.L., Hiroi, T., Meibom, A., Sunshine, J.M. 2000. Forging Asteroid-Meteorite Relationships Through Reflectance Spectroscopy. Lunar and Planetary Institute Science Conference Abstracts 31, 1844.

- Butler, R. P., and 10 colleagues 2006. Catalog of Nearby Exoplanets. The Astrophysical Journal 646, 505-522.

- Cameron, A. G. W. 1978. Physics of the primitive solar accretion disk. Moon and Planets 18, $5-40$.

- Canup, R. M., Esposito, L. W. 1996. Accretion of the Moon from an Impact-Generated Disk. Icarus 119, 427-446. 
- Canup, R. M., Asphaug, E. 2001. Origin of the Moon in a giant impact near the end of the Earth's formation. Nature 412, 708-712.

- Canup, R. M., Ward, W. R. 2006. A common mass scaling for satellite systems of gaseous planets. Nature 441, 834-839.

- Capobianco, C. C., Duncan, M., Levison, H. F. 2011. Planetesimal-driven planet migration in the presence of a gas disk. Icarus 211, 819-831.

- Carpenter, J. M., and 14 colleagues 2009. Formation and Evolution of Planetary Systems: Properties of Debris Dust Around Solar-Type Stars. The Astrophysical Journal Supplement Series 181, 197-226.

- Cassen, P. M., Smith, B. F., Miller, R. H., Reynolds, R. T. 1981. Numerical experiments on the stability of preplanetary disks. Icarus 48, 377-392.

- Chambers, J. E., Wetherill, G. W. 1998. Making the Terrestrial Planets: N-Body Integrations of Planetary Embryos in Three Dimensions. Icarus 136, 304-327.

- Chambers, J. E. 2001. Making More Terrestrial Planets. Icarus 152, 205-224.

- Chambers, J. E., Cassen, P. 2002. The effects of nebula surface density profile and giant-planet

- Chambers, J. 2006. A semi-analytic model for oligarchic growth. Icarus 180, 496-513.

- Chatterjee, S., Ford, E. B., Matsumura, S., Rasio, F. A. 2008. Dynamical Outcomes of PlanetPlanet Scattering. The Astrophysical Journal 686, 580-602.

- Chiang, E. I. 2003. Excitation of Orbital Eccentricities by Repeated Resonance Crossings: Requirements. The Astrophysical Journal 584, 465-471.

- Cohen, B. A., Swindle, T. D., Kring, D. A. 2000. Support for the Lunar Cataclysm Hypothesis from Lunar Meteorite Impact Melt Ages. Science 290, 1754-1756.

- Cresswell, P., Dirksen, G., Kley, W., Nelson, R. P. 2007. On the evolution of eccentric and inclined protoplanets embedded in protoplanetary disks. Astronomy and Astrophysics 473, 329-342.

- Crida, A., Morbidelli, A., Masset, F. 2006. On the width and shape of gaps in protoplanetary disks. Icarus 181, 587-604.

- Crida, A., Morbidelli, A. 2007. Cavity opening by a giant planet in a protoplanetary disc and effects on planetary migration. Monthly Notices of the Royal Astronomical Society 377, $1324-1336$.

- Crida, A., Sándor, Z., Kley, W. 2008. Influence of an inner disc on the orbital evolution of massive planets migrating in resonance. Astronomy and Astrophysics 483, 325-337. 
- Crida, A., Masset, F., Morbidelli, A. 2009. Long Range Outward Migration of Giant Planets, with Application to Fomalhaut b. The Astrophysical Journal 705, L148-L152.

- D'Angelo, G., Lubow, S. H., Bate, M. R. 2006. Evolution of Giant Planets in Eccentric Disks. The Astrophysical Journal 652, 1698-1714.

- Dauphas, N., Pourmand, A. 2011. Hf-W-Th evidence for rapid growth of Mars and its status as a planetary embryo. Nature 473, 489-492.

- Deutsch, A., Schaerer, U. 1994. Dating terrestrial impact events. Meteoritics 29, 301-322.

- di Sisto, R. P., Brunini, A. 2007. The origin and distribution of the Centaur population. Icarus 190, 224-235.

- Durisen, R. H., Boss, A. P., Mayer, L., Nelson, A. F., Quinn, T., Rice, W. K. M. 2007. Gravitational Instabilities in Gaseous Protoplanetary Disks and Implications for Giant Planet Formation. Protostars and Planets V 607-622.

- Fernandez, J. A., Ip, W.-H. 1984. Some dynamical aspects of the accretion of Uranus and Neptune - The exchange of orbital angular momentum with planetesimals. Icarus 58, 109-120.

- Ferraz-Mello, S., Beaugé, C., Michtchenko, T. A. 2003. Evolution of Migrating Planet Pairs in Resonance. Celestial Mechanics and Dynamical Astronomy 87, 99-112.

- Fischer, D. A., Valenti, J. 2005. The Planet-Metallicity Correlation. The Astrophysical Journal 622, 1102-1117.

- Fogg, M. J., Nelson, R. P. 2005. Oligarchic and giant impact growth of terrestrial planets in the presence of gas giant planet migration. Astronomy and Astrophysics 441, 791-806.

- Fogg, M. J., Nelson, R. P. 2007. On the formation of terrestrial planets in hot-Jupiter systems. Astronomy and Astrophysics 461, 1195-1208.

- Ford, E. B., Havlickova, M., Rasio, F. A. 2001. Dynamical Instabilities in Extrasolar Planetary Systems Containing Two Giant Planets. Icarus 150, 303-313.

- Ford, E. B., Rasio, F. A. 2008. Origins of Eccentric Extrasolar Planets: Testing the PlanetPlanet Scattering Model. The Astrophysical Journal 686, 621-636.

- Fouchet, T., Moses, J. I., Conrath, B. J. 2009. Saturn: Composition and Chemistry. Saturn from Cassini-Huygens 83.

- Fuentes, C. I., Holman, M. J. 2008. a SUBARU Archival Search for Faint Trans-Neptunian Objects. The Astronomical Journal 136, 83-97.

- Gáspár, A., Rieke, G. H., Su, K. Y. L., Balog, Z., Trilling, D., Muzzerole, J., Apai, D., Kelly, B. C. 2009. The Low Level of Debris Disk Activity at the Time of the Late Heavy Bombardment: A Spitzer Study of Praesepe. The Astrophysical Journal 697, 1578-1596. 
- Goldreich, P., Tremaine, S. 1979. The excitation of density waves at the Lindblad and corotation resonances by an external potential. The Astrophysical Journal 233, 857-871.

- Goldreich, P., Tremaine, S. 1980. Disk-satellite interactions. The Astrophysical Journal 241, 425-441.

- Goldreich, P., Sari, R. 2003. Eccentricity Evolution for Planets in Gaseous Disks. The Astrophysical Journal 585, 1024-1037.

- Goldreich, P., Lithwick, Y., Sari, R. 2004. Final Stages of Planet Formation. The Astrophysical Journal 614, 497-507.

- Gomes, R. S., Morbidelli, A., Levison, H. F. 2004. Planetary migration in a planetesimal disk: why did Neptune stop at 30 AU?. Icarus 170, 492-507.

- Gomes, R., Levison, H. F., Tsiganis, K., Morbidelli, A. 2005. Origin of the cataclysmic Late Heavy Bombardment period of the terrestrial planets. Nature 435, 466-469.

- Gradie, J., Tedesco, E. 1982. Compositional structure of the asteroid belt. Science 216, 1405-1407.

- Greenberg, R., Hartmann, W. K., Chapman, C. R., Wacker, J. F. 1978. Planetesimals to planets - Numerical simulation of collisional evolution. Icarus 35, 1-26.

- Greenzweig, Y., Lissauer, J. J. 1992. Accretion rates of protoplanets. II - Gaussian distributions of planetesimal velocities. Icarus 100, 440-463.

- Guillot, T. 2005. THE INTERIORS OF GIANT PLANETS: Models and Outstanding Questions. Annual Review of Earth and Planetary Sciences 33, 493-530.

- Guillot, T., Hueso, R. 2006. The composition of Jupiter: sign of a (relatively) late formation in a chemically evolved protosolar disc. Monthly Notices of the Royal Astronomical Society 367, L47-L51.

- Guillot, T., Santos, N. C., Pont, F., Iro, N., Melo, C., Ribas, I. 2006. A correlation between the heavy element content of transiting extrasolar planets and the metallicity of their parent stars. Astronomy and Astrophysics 453, L21-L24.

- Haisch, K. E., Jr., Lada, E. A., Lada, C. J. 2001. Disk Frequencies and Lifetimes in Young Clusters. The Astrophysical Journal 553, L153-L156.

- Hansen, B. M. S. 2009. Formation of the Terrestrial Planets from a Narrow Annulus. The Astrophysical Journal 703, 1131-1140.

- Hartmann, W. K., Ryder, G., Dones, L., Grinspoon, D. 2000. The Time-Dependent Intense Bombardment of the Primordial Earth/Moon System. Origin of the earth and moon, edited 
by R.M. Canup and K. Righter and 69 collaborating authors. Tucson: University of Arizona Press., p.493-512 493-512.

- Hartmann, W. K., Quantin, C., Mangold, N. 2007. Possible long-term decline in impact rates. 2. Lunar impact-melt data regarding impact history. Icarus 186, 11-23.

- Hayashi, C. 1981. Structure of the Solar Nebula, Growth and Decay of Magnetic Fields and Effects of Magnetic and Turbulent Viscosities on the Nebula. Progress of Theoretical Physics Supplement 70, 35-53.

- Henrard, J. 1993. The adiabatic invariants in classical mechanics, Dynamics Reported 2, $117-235$.

- Hillenbrand, L. A., Carpenter, J. M., Kim, J. S., Meyer, M. R., Backman, D. E., Moro-Martín, A., Hollenbach, D. J., Hines, D. C., Pascucci, I., Bouwman, J. 2008. The Complete Census of $70 \mu \mathrm{m}$-bright Debris Disks within "the Formation and Evolution of Planetary Systems" Spitzer Legacy Survey of Sun-like Stars. The Astrophysical Journal 677, 630-656.

- Ida, S., Makino, J. 1993. Scattering of planetesimals by a protoplanet - Slowing down of runaway growth. Icarus 106, 210.

- Ida, S., Bryden, G., Lin, D. N. C., Tanaka, H. 2000. Orbital Migration of Neptune and Orbital Distribution of Trans-Neptunian Objects. The Astrophysical Journal 534, 428-445.

- Ida, S., Lin, D. N. C. 2004. Toward a Deterministic Model of Planetary Formation. II. The Formation and Retention of Gas Giant Planets around Stars with a Range of Metallicities. The Astrophysical Journal 616, 567-572.

- Ida, S., Lin, D. N. C. 2008. Toward a Deterministic Model of Planetary Formation. V. Accumulation Near the Ice Line and Super-Earths. The Astrophysical Journal 685, 584-595.

- Jurić, M., Tremaine, S. 2008. Dynamical Origin of Extrasolar Planet Eccentricity Distribution. The Astrophysical Journal 686, 603-620.

- Kalas, P., Graham, J. R., Chiang, E., Fitzgerald, M. P., Clampin, M., Kite, E. S., Stapelfeldt, K., Marois, C., Krist, J. 2008. Optical Images of an Exosolar Planet 25 Light-Years from Earth. Science 322, 1345.

- Kelsall, T., and 11 colleagues 1998. The COBE Diffuse Infrared Background Experiment Search for the Cosmic Infrared Background. II. Model of the Interplanetary Dust Cloud. The Astrophysical Journal 508, 44-73.

- Kenyon, S. J., Bromley, B. C. 2006. Terrestrial Planet Formation. I. The Transition from Oligarchic Growth to Chaotic Growth. The Astronomical Journal 131, 1837-1850. 
- Kenyon, S. J., Bromley, B. C., O’Brien, D. P., Davis, D. R. 2008. Formation and Collisional Evolution of Kuiper Belt Objects. The Solar System Beyond Neptune 293-313.

- Kirsh, D. R., Duncan, M., Brasser, R., Levison, H. F. 2009. Simulations of planet migration driven by planetesimal scattering. Icarus 199, 197-209.

- Kleine, T., Touboul, M., Bourdon, B., Nimmo, F., Mezger, K., Palme, H., Jacobsen, S. B., Yin, Q.-Z., Halliday, A. N. 2009. Hf-W chronology of the accretion and early evolution of asteroids and terrestrial planets. Geochimica et Cosmochimica Acta 73, 5150-5188.

- Kley, W., Peitz, J., Bryden, G. 2004. Evolution of planetary systems in resonance. Astronomy and Astrophysics 414, 735-747.

- Kley, W., Lee, M. H., Murray, N., Peale, S. J. 2005. Modeling the resonant planetary system GJ 876. Astronomy and Astrophysics 437, 727-742.

- Kley, W., Dirksen, G. 2006. Disk eccentricity and embedded planets. Astronomy and Astrophysics 447, 369-377.

- Kley, W., Crida, A. 2008. Migration of protoplanets in radiative discs. Astronomy and Astrophysics 487, L9-L12.

- Koeberl, C. 2004. The late heavy bombardment in the inner solar system: is there any connection to Kuiper belt objects? The first decadal review of the Edgeworth-Kuiper Belt. $79-87$.

- Kokubo, E., Ida, S. 1998. Oligarchic Growth of Protoplanets. Icarus 131, 171-178.

- Kokubo, E., Kominami, J., Ida, S. 2006. Formation of Terrestrial Planets from Protoplanets. I. Statistics of Basic Dynamical Properties. The Astrophysical Journal 642, 1131-1139.

- Kokubo, E., Genda, H. 2010. Formation of Terrestrial Planets from Protoplanets Under a Realistic Accretion Condition. The Astrophysical Journal 714, L21-L25.

- Kring, D. A., Cohen, B. A. 2002. Cataclysmic bombardment throughout the inner solar system 3.9-4.0 Ga. Journal of Geophysical Research (Planets) 107, 5009.

- Levison, H. F., Lissauer, J. J., Duncan, M. J. 1998. Modeling the Diversity of Outer Planetary Systems. The Astronomical Journal 116, 1998-2014.

- Levison, H. F., Dones, L., Chapman, C. R., Stern, S. A., Duncan, M. J., Zahnle, K. 2001. Could the Lunar "Late Heavy Bombardment" Have Been Triggered by the Formation of Uranus and Neptune?. Icarus 151, 286-306.

- Levison, H. F., Agnor, C. 2003. The Role of Giant Planets in Terrestrial Planet Formation. The Astronomical Journal 125, 2692-2713. 
- Levison, H. F., Morbidelli, A. 2007. Models of the collisional damping scenario for ice-giant planets and Kuiper belt formation. Icarus 189, 196-212.

- Levison, H. F., Morbidelli, A., Gomes, R., Backman, D. 2007. Planet Migration in Planetesimal Disks. Protostars and Planets V 669-684.

- Levison, H. F., Morbidelli, A., Vanlaerhoven, C., Gomes, R., Tsiganis, K. 2008. Origin of the structure of the Kuiper belt during a dynamical instability in the orbits of Uranus and Neptune. Icarus 196, 258-273.

- Levison, H. F., Bottke, W. F., Gounelle, M., Morbidelli, A., Nesvorný, D., Tsiganis, K. 2009. Contamination of the asteroid belt by primordial trans-Neptunian objects. Nature 460, 364-366.

- Levison, H. F., Thommes, E., Duncan, M. J. 2010. Modeling the Formation of Giant Planet Cores. I. Evaluating Key Processes. The Astronomical Journal 139, 1297-1314.

- Levison, H. F., Morbidelli, A., Tsiganis, K., Nesvorny, D. and Gomes, R. 2011. Late orbital instabilities in the outer planets induced by interaction with a self-gravitating planetesimal disk. To be submitted.

- Lin, D. N. C., Papaloizou, J. 1986a. On the tidal interaction between protoplanets and the primordial solar nebula. II - Self-consistent nonlinear interaction. The Astrophysical Journal 307, 395-409.

- Lin, D. N. C., Papaloizou, J. 1986. On the tidal interaction between protoplanets and the protoplanetary disk. III - Orbital migration of protoplanets. The Astrophysical Journal 309, 846-857.

- Lin, D. N. C., Bodenheimer, P., Richardson, D. C. 1996. Orbital migration of the planetary companion of 51 Pegasi to its present location. Nature 380, 606-607.

- Lin, D. N. C., Ida, S. 1997. On the Origin of Massive Eccentric Planets. The Astrophysical Journal 477, 781.

- Lodders, K. 2003. Solar System Abundances and Condensation Temperatures of the Elements. The Astrophysical Journal 591, 1220-1247.

- Lynden-Bell, D., Pringle, J. E. 1974. The evolution of viscous discs and the origin of the nebular variables.. Monthly Notices of the Royal Astronomical Society 168, 603-637.

- Lyra, W., Johansen, A., Klahr, H., Piskunov, N. 2009a. Standing on the shoulders of giants. Trojan Earths and vortex trapping in low mass self-gravitating protoplanetary disks of gas and solids. Astronomy and Astrophysics 493, 1125-1139. 
- Lyra, W., Johansen, A., Zsom, A., Klahr, H., Piskunov, N. 2009b. Planet formation bursts at the borders of the dead zone in 2D numerical simulations of circumstellar disks. Astronomy and Astrophysics 497, 869-888.

- Lyra, W., Paardekooper, S.-J., Mac Low, M.-M. 2010. Orbital Migration of Low-mass Planets in Evolutionary Radiative Models: Avoiding Catastrophic Infall. The Astrophysical Journal 715, L68-L73.

- Malhotra, R. 1993. The origin of Pluto's peculiar orbit. Nature 365, 819-821.

- Malhotra, R. 1995. The Origin of Pluto's Orbit: Implications for the Solar System Beyond Neptune. The Astronomical Journal 110, 420.

- Marois, C., Macintosh, B., Barman, T., Zuckerman, B., Song, I., Patience, J., Lafrenière, D., Doyon, R. 2008. Direct Imaging of Multiple Planets Orbiting the Star HR 8799. Science 322, 1348.

- Marzari, F., Weidenschilling, S. J. 2002. Eccentric Extrasolar Planets: The Jumping Jupiter Model. Icarus 156, 570-579.

- Marzari, F., Baruteau, C., Scholl, H. 2010. Planet-planet scattering in circumstellar gas disks. Astronomy and Astrophysics 514, L4.

- Masset, F., Snellgrove, M. 2001. Reversing type II migration: resonance trapping of a lighter giant protoplanet. Monthly Notices of the Royal Astronomical Society 320, L55-L59.

- Masset, F. S., Morbidelli, A., Crida, A., Ferreira, J. 2006. Disk Surface Density Transitions as Protoplanet Traps. The Astrophysical Journal 642, 478-487.

- Milani, A., Nobili, A. M., Carpino, M. 1987. Secular variations of the semimajor axes - Theory and experiments. Astronomy and Astrophysics 172, 265-279.

- Militzer, B., Hubbard, W. B. 2009. Comparison of Jupiter interior models derived from firstprinciples simulations. Astrophysics and Space Science 322, 129-133.

- Min, M., Dullemond, C. P., Kama, M., Dominik, C. 2011. The thermal structure and the location of the snow line in the protosolar nebula: Axisymmetric models with full 3-D radiative transfer. Icarus 212, 416-426.

- Minton, D. A., Malhotra, R. 2009. A record of planet migration in the main asteroid belt. Nature 457, 1109-1111.

- Moekel, N., Raymond, S.N., Armitage, Ph.J. 2008. Extrasolar planets eccentricities from scattering in the presence of residual gas-disks. Ap.J. 688, 1361-1367

- Moorhead, A. V., Adams, F. C. 2005. Giant planet migration through the action of disk torques and planet planet scattering. Icarus 178, 517-539. 
- Morbidelli, A., Chambers, J., Lunine, J. I., Petit, J. M., Robert, F., Valsecchi, G. B., Cyr, K. E. 2000. Source regions and time scales for the delivery of water to Earth. Meteoritics and Planetary Science 35, 1309-1320.

- Morbidelli, A., Crida, A. 2007. The dynamics of Jupiter and Saturn in the gaseous protoplanetary disk. Icarus 191, 158-171.

- Morbidelli, A., Tsiganis, K., Crida, A., Levison, H. F., Gomes, R. 2007. Dynamics of the Giant Planets of the Solar System in the Gaseous Protoplanetary Disk and Their Relationship to the Current Orbital Architecture. The Astronomical Journal 134, 1790-1798.

- Morbidelli, A., Crida, A., Masset, F., Nelson, R. P. 2008. Building giant-planet cores at a planet trap. Astronomy and Astrophysics 478, 929-937.

- Morbidelli, A., Levison, H. F., Gomes, R. 2008b. The Dynamical Structure of the Kuiper Belt and Its Primordial Origin. The Solar System Beyond Neptune 275-292.

- Morbidelli, A., Brasser, R., Gomes, R., Levison H.F. and Tsiganis, K., 2010. Evidence from the asteroid belt for a violent past evolution of Jupiter's orbit. Astron. J., submitted.

- Morishima, R., Stadel, J., Moore, B. 2010. From planetesimals to terrestrial planets: N-body simulations including the effects of nebular gas and giant planets. Icarus 207, 517-535.

- Morfill, G. E., Voelk, H. J. 1984. Transport of dust and vapor and chemical fractionation in the early protosolar cloud. The Astrophysical Journal 287, 371-395.

- Nelson, R. P., Papaloizou, J. C. B. 2003. The interaction of a giant planet with a disc with MHD turbulence - II. The interaction of the planet with the disc. Monthly Notices of the Royal Astronomical Society 339, 993-1005.

- Nelson, R. P. 2005. On the orbital evolution of low mass protoplanets in turbulent, magnetised disks. Astronomy and Astrophysics 443, 1067-1085.

- Nesvorný, D., Jenniskens, P., Levison, H. F., Bottke, W. F., Vokrouhlický, D., Gounelle, M. 2010. Cometary Origin of the Zodiacal Cloud and Carbonaceous Micrometeorites. Implications for Hot Debris Disks. The Astrophysical Journal 713, 816-836.

- Nettelmann, N., Holst, B., Kietzmann, A., French, M., Redmer, R., Blaschke, D. 2008. Ab Initio Equation of State Data for Hydrogen, Helium, and Water and the Internal Structure of Jupiter. The Astrophysical Journal 683, 1217-1228.

- O'Brien, D. P., Morbidelli, A., Levison, H. F. 2006. Terrestrial planet formation with strong dynamical friction. Icarus 184, 39-58.

- O'Brien, D. P., Morbidelli, A., Bottke, W. F. 2007. The primordial excitation and clearing of the asteroid belt - Revisited. Icarus 191, 434-452. 
- Öpik, E. J., 1976. Interplanetary Encounters: Close Range Gravitational Interactions. Elsevier, New York.

- Paardekooper, S.-J., Mellema, G. 2006. Halting type I planet migration in non-isothermal disks. Astronomy and Astrophysics 459, L17-L20.

- Paardekooper, S.-J., Papaloizou, J. C. B. 2009. On corotation torques, horseshoe drag and the possibility of sustained stalled or outward protoplanetary migration. Monthly Notices of the Royal Astronomical Society 394, 2283-2296.

- Paardekooper, S.-J., Baruteau, C., Crida, A., Kley, W. 2010. A torque formula for nonisothermal type I planetary migration - I. Unsaturated horseshoe drag. Monthly Notices of the Royal Astronomical Society 401, 1950-1964.

- Papaloizou, J. C. B., Nelson, R. P., Masset, F. 2001. Orbital eccentricity growth through disc-companion tidal interaction. Astronomy and Astrophysics 366, 263-275.

- Petit, J.-M., Morbidelli, A., Chambers, J. 2001. The Primordial Excitation and Clearing of the Asteroid Belt. Icarus 153, 338-347.

- Pierens, A., Nelson, R. P. 2008. Constraints on resonant-trapping for two planets embedded in a protoplanetary disc. Astronomy and Astrophysics 482, 333-340.

- Podolak, M., Zucker, S. 2004. A note on the snow line in protostellar accretion disks. Meteoritics and Planetary Science 39, 1859-1868.

- Pollack, J. B., Hubickyj, O., Bodenheimer, P., Lissauer, J. J., Podolak, M., Greenzweig, Y. 1996. Formation of the Giant Planets by Concurrent Accretion of Solids and Gas. Icarus 124, $62-85$.

- Rafikov, R. R. 2004. Fast Accretion of Small Planetesimals by Protoplanetary Cores. The Astronomical Journal 128, 1348-1363.

- Rasio, F. A., Ford, E. B. 1996. Dynamical instabilities and the formation of extrasolar planetary systems. Science 274, 954-956.

- Raymond, S. N., Quinn, T., Lunine, J. I. 2004. Making other earths: dynamical simulations of terrestrial planet formation and water delivery. Icarus 168, 1-17.

- Raymond, S. N., Quinn, T., Lunine, J. I. 2005. Terrestrial Planet Formation in Disks with Varying Surface Density Profiles. The Astrophysical Journal 632, 670-676.

- Raymond, S. N., Quinn, T., Lunine, J. I. 2006. High-resolution simulations of the final assembly of Earth-like planets I. Terrestrial accretion and dynamics. Icarus 183, 265-282.

- Raymond, S. N., Mandell, A. M., Sigurdsson, S. 2006b. Exotic Earths: Forming Habitable Worlds with Giant Planet Migration. Science 313, 1413-1416. 
- Raymond, S. N., Quinn, T., Lunine, J. I. 2007. High-Resolution Simulations of The Final Assembly of Earth-Like Planets. 2. Water Delivery And Planetary Habitability. Astrobiology 7, 66-84.

- Raymond, S. N., Barnes, R., Veras, D., Armitage, P. J., Gorelick, N., Greenberg, R. 2009. Planet-Planet Scattering Leads to Tightly Packed Planetary Systems. The Astrophysical Journal 696, L98-L101.

- Raymond, S. N., O’Brien, D. P., Morbidelli, A., Kaib, N. A. 2009b. Building the terrestrial planets: Constrained accretion in the inner Solar System. Icarus 203, 644-662.

- Ryder, G., 1990, Lunar samples, lunar accretion and the Early bombardment of the Moon: Eos Transactions AGU, v. 71, p. 313-323.

- Ryder, G., Koeberl, C., and Mojzsis, S.J., 2000, Heavy bombardment on the Earth at 3.85: The search for petrographical and geochemical evidence, in Canup, R.M., and Righter, K., eds., Origin of the Earth and Moon: Tucson, Arizona, University of Arizona Press, p. 475-492.

- Ryder, G., 2002, Mass flux in the ancient Earth-Moon system and the benign implications for the origin of life on Earth: Journal Geophysical Research-Planets, v. 107, p. 6-14.

- Sándor, Z., Lyra, W., Dullemond, C. P. 2011. Formation of Planetary Cores at Type I Migration Traps. The Astrophysical Journal 728, L9.

- Saslaw, W. C. 1985. Thermodynamics and galaxy clustering - Relaxation of N-body experiments. The Astrophysical Journal 297, 49-60.

- Stamatellos, D., Whitworth, A. P. 2008. Can giant planets form by gravitational fragmentation of discs? Astronomy and Astrophysics 480, 879-887.

- Stewart, G., Wetherill, G. 1988. Evolution of planetesimal velocities. Icarus 79, 542-553.

- Shakura, N.I. and Sunyaev, R.A. 1973. Black holes in binary systems. Observational appearance. Astronomy and Astrophysics 24, 337-355.

- Tanaka, H., Takeuchi, T., Ward, W. R. 2002. Three-Dimensional Interaction between a Planet and an Isothermal Gaseous Disk. I. Corotation and Lindblad Torques and Planet Migration. The Astrophysical Journal 565, 1257-1274.

- Tera, F., Papanastassiou, D. A., Wasserburg, G. J. 1974. Isotopic evidence for a terminal lunar cataclysm. Earth and Planetary Science Letters 22, 1.

- Thommes, E. W., Duncan, M. J., Levison, H. F. 1999. The formation of Uranus and Neptune in the Jupiter-Saturn region of the Solar System. Nature 402, 635-638.

- Thommes, E. W., Duncan, M. J., Levison, H. F. 2003. Oligarchic growth of giant planets. Icarus 161, 431-455. 
- Thommes, E., Nagasawa, M., Lin, D. N. C. 2008. Dynamical Shake-up of Planetary Systems. II. N-Body Simulations of Solar System Terrestrial Planet Formation Induced by Secular Resonance Sweeping. The Astrophysical Journal 676, 728-739.

- Tiscareno, M. S., Malhotra, R. 2003. The Dynamics of Known Centaurs. The Astronomical Journal 126, 3122-3131.

- Trail, D., Mojzsis, S. J., Harrison, T. M. 2007. Thermal events documented in Hadean zircons by ion microprobe depth profiles. Geochimica et Cosmochimica Acta 71, 4044-4065.

- Tsiganis, K., Gomes, R., Morbidelli, A., Levison, H. F. 2005. Origin of the orbital architecture of the giant planets of the Solar System. Nature 435, 459-461.

- Valley J. W., Peck W. H., King E. M., and Wilde S. A. 2002. A cool early Earth. Geology $30,351-354$.

- Valsecchi, A., Manara, G. B. 1997. Dynamics of comets in the outer planetary region. II. Enhanced planetary masses and orbital evolutionary paths. Astronomy and Astrophysics 323, 986-998.

- Veras, D., Armitage, P. J. 2004. Outward migration of extrasolar planets to large orbital radii. Monthly Notices of the Royal Astronomical Society 347, 613-624.

- Veras, D., Crepp, J. R., Ford, E. B. 2009. Formation, Survival, and Detectability of Planets Beyond 100 AU. The Astrophysical Journal 696, 1600-1611.

- Walsh, K.J., Morbidelli, A., Raymond, S.N. O'Brien, D.P., Mandell, A.M. 2011. A low mass for Mars from Jupiter's early gas-driven migration. Nature, in press.

- Ward, W. R. 1986. Density waves in the solar nebula - Differential Lindblad torque. Icarus 67, 164-180.

- Ward, W. R. 1997. Protoplanet Migration by Nebula Tides. Icarus 126, 261-281.

- Weidenschilling, S. J. 1977. The distribution of mass in the planetary system and solar nebula. Astrophysics and Space Science 51, 153-158.

- Weidenschilling, S. J., Marzari, F. 1996. Gravitational scattering as a possible origin for giant planets at small stellar distances. Nature 384, 619-621.

- Weidenschilling, S. J., Spaute, D., Davis, D. R., Marzari, F., Ohtsuki, K. 1997. Accretional Evolution of a Planetesimal Swarm. Icarus 128, 429-455.

- Wetherill, G. W., Stewart, G. R. 1989. Accumulation of a swarm of small planetesimals. Icarus 77, 330-357. 
- Wetherill, G. W. 1992. An alternative model for the formation of the asteroids. Icarus 100, 307-325.

- Wetherill, G. W., Stewart, G. R. 1993. Formation of planetary embryos - Effects of fragmentation, low relative velocity, and independent variation of eccentricity and inclination. Icarus 106, 190.

- Villeneuve, J., Chaussidon, M., Libourel, G. 2009. Homogeneous Distribution of 26Al in the Solar System from the Mg Isotopic Composition of Chondrules. Science 325, 985-988.

- Wong, M. H., Mahaffy, P. R., Atreya, S. K., Niemann, H. B., Owen, T. C. 2004. Updated Galileo probe mass spectrometer measurements of carbon, oxygen, nitrogen, and sulfur on Jupiter. Icarus 171, 153-170.

- Wyatt, M. C., Smith, R., Greaves, J. S., Beichman, C. A., Bryden, G., Lisse, C. M. 2007. Transience of Hot Dust around Sun-like Stars. The Astrophysical Journal 658, 569-583.

- Zhang, H., Zhou, J.-L. 2010. On the Orbital Evolution of a Giant Planet Pair Embedded in a Gaseous Disk. I. Jupiter-Saturn Configuration. The Astrophysical Journal 714, 532-548. 


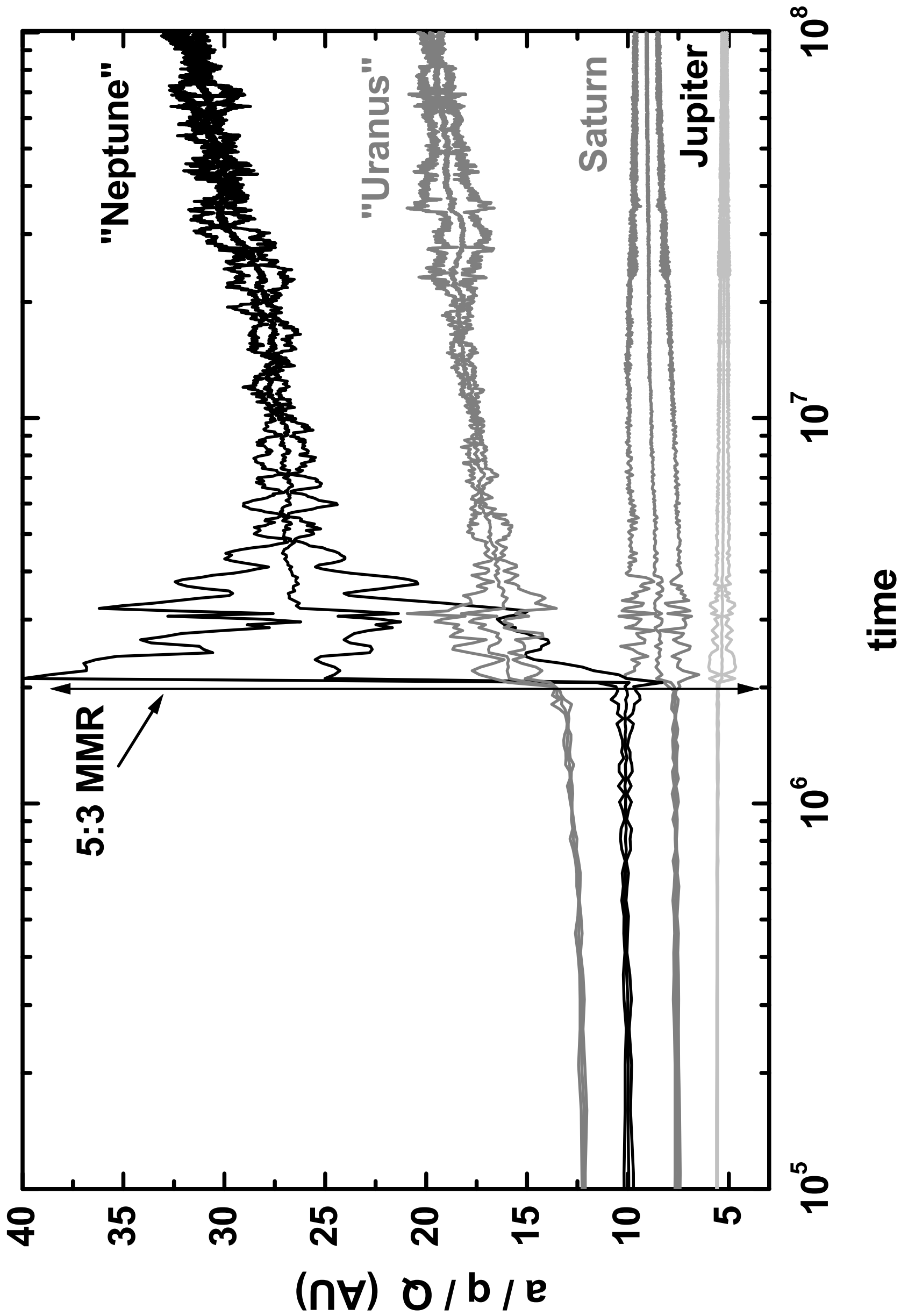

